\title{
Host lung environment limits Aspergillus fumigatus germination through a SskA-dependent signaling response
}

Marina E. Kirkland ${ }^{1}$, McKenzie Stannard ${ }^{1}$, Caitlin H. Kowalski ${ }^{1}$, Dallas Mould ${ }^{1}$, Alayna CaffreyCarr $^{1,2}$, Rachel M. Temple ${ }^{1}$, Brandon S. Ross ${ }^{1}$, Lotus A. Lofgren ${ }^{3}$, Jason E. Stajich ${ }^{3}$, Robert A. Cramer ${ }^{1}$, Joshua J. Obar ${ }^{1, \#}$

${ }^{1}$ Geisel School of Medicine at Dartmouth, Department of Microbiology \& Immunology, Lebanon, $\mathrm{NH}$, United States

${ }^{2}$ Montana State University, Department of Microbiology \& Immunology, Bozeman, MT, United States

${ }^{3}$ Department of Microbiology \& Plant Pathology and Institute for Integrative Genome Biology, University of California Riverside, Riverside, CA, United States

Running Title: Aspergillus growth in the lungs is regulated by SakA

Conflict of Interests: The authors have declared that no conflict of interest exists.

\#Corresponding author:

Joshua J. Obar

Geisel School of Medicine at Dartmouth

Department of Microbiology \& Immunology

1 Medical Center Drive

Lebanon, NH 03756

Telephone: +1 (603) 646-5384

Email: joshua.j.obar@dartmouth.edu 


\section{ABSTRACT}

Aspergillus fumigatus isolates display significant heterogeneity in growth, virulence, pathology, and inflammatory potential in multiple murine models of invasive aspergillosis. Previous studies have linked the initial germination of a fungal isolate in the airways to the inflammatory and pathological potential; but the mechanism(s) regulating A. fumigatus germination in the airways are unresolved. To explore the genetic basis for divergent germination phenotypes, we utilized a serial passaging strategy in which we cultured a slow germinating strain (AF293) in a murine lung based medium for multiple generations. Through this serial passaging approach, a strain emerged with an increased germination rate that induces more inflammation than the parental strain (herein

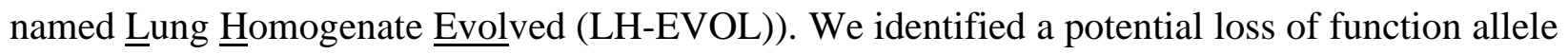
of Afu5g08390 (sskA) in the LH-EVOL strain. The LH-EVOL strain had a decreased ability to induce the SakA-dependent stress pathway, similar to AF293 $\Delta s s k A$ and CEA10. In support of the whole genome variant analyses, $s s k A$, sakA, or $m p k C$ loss of function strains in the AF293 parental strain increased germination both in vitro and in vivo. Since the airway surface liquid of the lungs contains low glucose levels, the relationship of low glucose concentration on germination of these mutant AF293 strains was examined; interestingly, in low glucose conditions the sakA pathway mutants exhibited an enhanced germination rate. In conclusion, A. fumigatus germination in the airways is regulated by SskA through the SakA MAPK pathway and drives enhanced disease initiation and inflammation in the lungs. 


\section{IMPORTANCE}

Aspergillus fumigatus is an important human fungal pathogen particularly in immunocompromised individuals. Initiation of growth by A. fumigatus in the lung is important for its pathogenicity in murine models. However, our understanding of what regulates fungal germination in the lung environment is lacking. Through a serial passage experiment using lung-based medium, we identified a new strain of $A$. fumigatus which has increased germination potential and inflammation in the lungs. Using this serially passaged strain we found it had a decreased ability to mediate signaling through the osmotic stress response pathway. This finding was confirmed using genetic null mutants demonstrating that the osmotic stress response pathway is critical for regulating growth in the murine lungs. Our results contribute to the understanding of A. fumigatus adaptation and growth in the host lung environment. 


\section{INTRODUCTION}

Aspergillus fumigatus is a ubiquitous mold essential for decomposition of organic material that can easily become airborne. Infection is established in the respiratory tract by inhalation of dormant conidia which are roughly $2 \mu \mathrm{m}$ in diameter. Their small size facilitates embedment into tissues if those resting conidia are not removed through mucociliary action of respiratory epithelial cells. If allowed sufficient time, A. fumigatus conidia will begin to germinate, which initiates with osmotic swelling and then polarized growth of germ tube. At this point, a healthy immune system can detect pathogen associated molecular patterns (PAMPs) on the cell surface of swollen or germinated conidia, clearing the swollen conidia before disease can be established. Should this occur in an immunocompromised host, germination can go unchecked resulting in fungal biofilm formation and tissue invasion establishing severe disease ${ }^{1-3}$.

Previous work has shown a correlation between germination and pathogenicity of $A$. fumigatus in a strain-dependent manner in a model of bronchopneumonia ${ }^{4}$. Between clinical isolates AF293 and CEA10, CEA10 exhibits faster germination both in vitro and in vivo in murine lung airways ${ }^{4}$, as well as in a zebrafish infection model $^{5}$. This increased germination corresponds with greater tissue damage and greater inflammation following CEA10 challenge compared to AF293 challenge ${ }^{4-6}$. However, conditions and genetic pathways that regulate the germination rate for each strain in the lung microenvironment remain unresolved.

For germination to occur conidia must first sense whether their environment has sufficient nutrient availability and the presence of potential stressors. In Aspergillus nidulans, the presence of glucose was sufficient to induce germination of conidia ${ }^{7}$, while in A. fumigatus glucose in the presence of water and oxygen to drive cellular respiration was necessary for conidia germination ${ }^{8}$. The germination process is regulated by both Ras signaling ${ }^{9}$ and G-protein signaling through the 
cAMP-PKA signaling pathway ${ }^{8-10}$. Additionally, calcium signaling through the calcineurin pathway and the CrzA transcription factor is needed for A. fumigatus germination ${ }^{11,12}$. Further, in A. fumigatus nitrogen sources, such as ammonium chloride or proline, drive more robust germination in a SakA-regulated manner ${ }^{13}$. Once conidia begin to germinate, they begin an isotropic growth phase which modifies the composition of their cell walls, subjecting conidia to detection by a host's immune system. Polarized growth follows as swollen conidia develop into germlings then hyphae before establishing a complex network called mycelia ${ }^{1}$.

The lung itself has evolved to interact constantly with an onslaught of foreign particulate matter and microbes in the air. Lung airways are a low-nutrient environment: airway surface liquid (ASL) glucose concentrations in a healthy adult are approximately $0.4 \mathrm{~m} M$, which is about 10 -fold less than in the plasma ${ }^{14}$. Reduced nutrient availability is thought to limit microbial growth in the lung environment. In addition, the ASL facilitates the first line defense of airway epithelial cells by providing mucous and water secretions which assist in mucociliary clearance ${ }^{14-16}$. The ASL can become dysregulated in chronic disease states brought on by asthma ${ }^{17,18}, \mathrm{COPD}^{18}$, cystic fibrosis $^{19}$, and type II diabetes ${ }^{19-21}$ resulting in increased glucose levels ${ }^{21}$, all of which have increased risk for developing aspergillosis ${ }^{22-25}$.

In this study, we explored the molecular mechanisms regulating the germination of $A$. fumigatus in context of the murine lung environment. To do this we utilized a serial passaging approach by culturing the slow germinating AF293 strain in lung homogenate medium and selecting for increased germination after multiple generations. A novel AF293-dervived strain, LH-EVOL, was selected and found to germinate quicker in the murine lung airways compared to the parental AF293 strain resulting in a stronger proinflammatory IL-1 $\alpha$-dependent immune response. Whole genome sequencing of LH-EVOL revealed conserved mutations in Afu5g08390 
$(s s k A)$, which is part of the SakA MAPK stress pathway ${ }^{26}$. In vitro and in vivo growth kinetics of an AF293 $\Delta s s k A$ mutant recapitulated the quick germinating phenotype of LH-EVOL. Interestingly, this rapid germination phenotype was further accelerated in vitro under low glucose conditions, which more closely resemble the concentration of glucose found in the lung environment. Taken together, this study further supports previous observations that germination rate is a key step in determining the qualitative and quantitative inflammatory immune response to A. fumigatus strains in the murine lung and identifies the SakA-pathway as a key mediator of host lung environment. 


\section{MATERIALS AND METHODS}

Fungal strains and growth conditions. All fungal strains used in this study are listed in Supplemental Table 1. For conidial harvest, strains were cultured on $1 \%$ glucose minimal media (GMM; $1 \%$ or $55.56 \mathrm{~m} M$ glucose, $6 \mathrm{~g} / \mathrm{L} \mathrm{NaNO}_{3}, 0.52 \mathrm{~g} / \mathrm{L} \mathrm{KCl}, 0.52 \mathrm{~g} / \mathrm{L} \mathrm{MgSO}{ }_{4} \cdot 7 \mathrm{H}_{2} \mathrm{O}, 1.52 \mathrm{~g} / \mathrm{L}$ $\mathrm{KH}_{2} \mathrm{PO}_{4}$ monobasic, $2.2 \mathrm{mg} / \mathrm{L} \mathrm{ZnSO}_{4} \bullet 7 \mathrm{H}_{2} \mathrm{O}, 1.1 \mathrm{mg} / \mathrm{L} \mathrm{H}_{3} \mathrm{BO}_{3}, 0.5 \mathrm{mg} / \mathrm{L} \mathrm{MnCl}{ }_{2} \bullet 4 \mathrm{H}_{2} \mathrm{O}, 0.5 \mathrm{mg} / \mathrm{L}$ $\mathrm{FeSO}_{4} \bullet 7 \mathrm{H}_{2} \mathrm{O}, 0.16 \mathrm{mg} / \mathrm{L} \mathrm{CoCl}_{2} \bullet 5 \mathrm{H}_{2} \mathrm{O}, 0.16 \mathrm{mg} / \mathrm{L} \mathrm{CuSO}_{4} \cdot 5 \mathrm{H}_{2} \mathrm{O}, 0.11 \mathrm{mg} / \mathrm{L}\left(\mathrm{NH}_{4}\right)_{6} \mathrm{Mo}_{7} \mathrm{O}_{24} \bullet 4 \mathrm{H}_{2} \mathrm{O}$, and $5 \mathrm{mg} / \mathrm{L} \mathrm{Na}$ EDTA; $\mathrm{pH}$ 6.5) agar plates for three days at $37^{\circ} \mathrm{C}$, at which time conidia were collected by flooding plates with $0.01 \%$ PBS/Tween 80 and gently scraping mycelia using a cell scraper. Conidia were then filtered through sterile Miracloth, washed, and resuspended in phosphate buffered saline (PBS), and counted on a hemacytometer. Strains were stored in PBS and were used immediately for in vitro experiments or allowed to rest overnight for in vivo studies.

Serial passaging of AF293 through murine lung homogenate medium. The wild-type AF293 strain of A. fumigatus was used for a serial passage experiment as it germinates slowly in the lung airways $^{6}$. Murine lung homogenate medium was made as previously described ${ }^{6}$, where naïve C57BL/6J lungs were crushed in $2 \mathrm{ml} \mathrm{PBS}$ and filtered through a $70 \mu \mathrm{M}$ filter after which cellular debris was removed by centrifugation. The working concentration used was 1:4 in PBS and contains $\sim 35 \mu \mathrm{M}$ glucose, which was determined using a glucose oxidase assay kit. AF293 was cultured in lung homogenate medium for 8 hours at $37^{\circ} \mathrm{C}$ shaking at $350 \mathrm{rpm}$ to induce germination. The fungal material was then collected and plated on 1\% GMM agar plates and grown for 3 days at $37^{\circ} \mathrm{C}$. The population of conidia were then collected and cataloged. This process was repeated 13 times. LH-EVOL was then grown under standard growth conditions prior to further 
analyses. Twenty individual clones of the LH-EVOL population were isolated to a single spore for further analysis in our lung homogenate germination assay.

Whole genome sequencing and variant identification. To assess potential mutations within the LHEVOL strain whole genome DNA sequencing was performed on three LH-EVOL clones (LHEVOL clone 4, LH-EVOL clone 11, and LH-EVOL clone 12) with enhanced germination in lung homogenate medium. Genomic DNA was extracted from mycelia grown in Petri plates using liquid $1 \%$ GMM supplemented with $0.5 \%$ yeast extract, following to previously published methods $^{27}$. The DNA concentration was quantified using a Qubit 2.0 Fluorometer (Invitrogen) and the manufacturer's recommended Broad Range protocol. The genomic DNA sequencing was genomic sequencing libraries were prepared by SeqMatic (Fremont, CA) using Illumina TruSeq DNA kits and sequenced on MiSeq Illumina sequencer in 2x250 bp format following manufacturer recommendations for paired end library construction and barcoding. The sequencing produced a range of $1-1$ average of $1.1 \mathrm{M}-1.6 \mathrm{M}$ reads, which is from $300-440 \mathrm{Mb}$ and $10-14 \mathrm{X}$ coverage of the A. fumigatus genome.

The DNA sequence reads for each strain were aligned to the AF293 reference genome downloaded from FungiDB v.39 28,29 with BWA v0.7.17 30 and converted to BAM files using SAMtools $\mathrm{v} 1.10^{31}$. Following best practices $^{32}$, the reads were marked for PCR and optical duplication using picard tools v2.18.3 (http://broadinstitute.github.io/picard). For variants identified near inferred INDELs, reads were realigned using RealignerTargetCreator and IndelRealigner in the Genome Analysis Toolkit GATK v3.7². The SNPs and INDELS were genotyped relative to AF293 using HaplotypeCaller in GATK v4.1.1.0 ${ }^{33}$. Filtering was accomplished with GATK's SelectVariants with the following parameters: for SNPS: -window- 
size $=10,-$ QualByDept $<2.0$, -MapQual $<40.0$, -QScore $<100,-$ MapQualityRankSum $<-12.5$, -StrandOddsRatio > 3.0, -FisherStrandBias > 60.0, -ReadPosRankSum < -8.0. For INDELS: window-size $=10$, -QualByDepth $<2.0,-$ MapQualityRankSum $<-12.5,-$ StrandOddsRatio $>4.0$, -FisherStrandBias $>200.0$, -ReadPosRank $<-20.0$, -InbreedingCoeff $<-0.8$. The genic overlap and consequence of the variants were classified with snpEff ${ }^{34}$. Variants were transformed into tabular format from VCF format using script 'snpEff_2_tab.py'.

The genomic short reads for the four strains are deposited in NCBI SRA associated with BioProject PRJNA757188. Scripts for running the pipeline for analyses are available in the github (https://github.com/stajichlab/Afum_LH-EVOL).

Aspergillus fumigatus pulmonary challenge model. C57BL/6J mice (Stock \#000664) were purchased from Jackson Laboratory at 7-8 weeks of age. Ill $a^{(-/-)}$mice $^{35}$ were bred in-house at Dartmouth College. Animal studies were carried out in accordance with the recommendations in the Guide for the Care and Use of Laboratory Animals. The animal experimental protocol 00002168 was approved by the Institutional Animal Care and Use Committee (IACUC) at Dartmouth College. Mice were challenged with $4 \times 10^{7}$ conidia in PBS intratracheally under isoflurane anesthesia in a volume of $100 \mu \mathrm{l}$. At the indicated experimental time points mice were euthanized and bronchoalveolar lavage fluid (BALF) was collected by washing the lungs with 2 $\mathrm{ml}$ of PBS containing 0.05 $M$ EDTA. BALF was clarified by centrifugation and stored at $-20^{\circ} \mathrm{C}$ until analysis. After centrifugation, the cellular component of the BAL was resuspended in $200 \mu \mathrm{l}$ of PBS. BAL cells were subsequently spun onto glass slides using a Cytospin4 cytocentrifuge (Thermo Scientific) and stained with Diff-Quik stain set (Siemens) for quantification of fungal germination, as previously described ${ }^{4}$. The percent germination of each A. fumigatus strain was 
quantified by manual counting of 100-200 fungal conidia and germlings at 100X magnification using a standard upright microscope. For histological analysis lungs were filled with and stored in $10 \%$ buffered formalin phosphate for at least 24 hours. Lungs were then embedded in paraffin and lung sections were stained with Grocott's methenamine silver (GMS) staining to assess fungal germination.

Murine lung RNA preparation and NanoString analysis. Animals were challenged with either PBS, AF293, or LH-EVOL, as described above. Forty hours after inoculation mice were euthanized and whole lungs were removed for mRNA analysis. Lungs were placed in $2 \mathrm{ml}$ of TRIzol and homogenized with a glass dounce homogenizer, followed by treatment with chloroform to extract RNA according to manufacturer's instructions. After RNA was assessed for quality, $100 \mathrm{ng}$ of RNA was used per reaction using the nCounter Mouse PanCancer Immune Profiling Panel (NanoString). nSolver 4.0 software was used for background subtraction and normalization. nSolver Advanced Analysis 2.0 was used for quality control analysis and pathway analysis.

Mutant Aspergillus fumigatus strain construction. The $\Delta s s k A$ strain was generated in the uracil/uridine auxotrophic strain AF293.1 through homologous recombination. The gene replacement construct was generated using overlap extension PCR as previously described ${ }^{36}$ in which $\sim 1 \mathrm{~kb}$ of sequence 5 ' to the start codon of $s s k A$ and $\sim 1 \mathrm{~kb} 3$ ' to stop codon of $s s k A$ were amplified and fused to Aspergillus parasiticus orotidine 5'-monophosphate decarboxylase gene, pyrG. This pyrG fragment was amplified from pSD38.1 where pyrG has been blunt-end ligated into pJET vector (Thermo Scientific, \#K1231) with 5' and 3' linker sequences, 5'accggtcgectcaaacaatgctct-3' and 5'- cgcatcagtgcctcctctcagac-3', respectively ${ }^{37}$. Primers for the 
amplification of this gene replacement construct are provided in Supplemental Table 2. The transformation of AF293.1 to generate $\Delta s s k A$ was carried out as previously described for the transformation of A. fumigatus protoplasts generated using Lysing Enzyme from Trichoderma harzianum (Sigma: L1412) ${ }^{38}$. Candidate $\Delta s s k A$ strains were selected for restored prototrophy. Single spores were isolated from the candidate strains to generate clonal strains that were confirmed to be $\triangle s s k A$ through PCR and Southern Blot analyses. Southern blot analyses were performed as previously described using the digoxigenin-anti-digoxigenin detection system $(\text { Roche Diagnostics) })^{38}$.

For the generation of the $s s k A^{R C}$ construct, the pBluescript II KS(+) (Addgene) was utilized. The multiple cloning site was expanded and the pyrithiamine resistance cassette was amplified from pPTR I (Takara) to include XhoI (New England Biolabs) restriction sites at the 5' and 3' ends and introduced via the SalI site (pSD11). From AF293 genomic DNA we amplified a $\sim 4 \mathrm{~kb}$ fragment that included $\sim 1 \mathrm{~kb} 5$ ' and $\sim 200 \mathrm{bp} \mathrm{3'}$ of $s s k A$ and introduced PacI and NotI (New England Biolabs) restriction sites at the 5' and 3' ends, respectively. The $s s k A^{R C}$ construct, pSskA$\mathrm{R}$ was confirmed through restriction digestion and Sanger sequencing. The construct was isolated (Zyppy Plasmid Miniprep, Zymo Research) and transformed into the $\Delta s s k A$ fungal genome as described above. The $s s k A^{R C}$ strain, which contained an ectopic insertion of the $s s k A$ wild-type allele, was confirmed with qRT-PCR as previously described ${ }^{37}$. The housekeeping genes utilized were $t u b 2$ and $a c t A$.

Fungal germination assays. In vitro fungal germination assays were conducted as previously described $^{6}$. Briefly, fungal strains were inoculated in either 1\% GMM (55.56 mM of glucose), $0.25 \%$ GMM (13.89 mM of glucose), 1\% GMM supplemented with $0.5 \%$ yeast extract (YE), or 
lung homogenate at a concentration of $1 \times 10^{7}$ conidia/ml in $2 \mathrm{ml}$ of medium in glass $20 \mathrm{ml}$ disposable scintillation vials (VWR). Cultures were incubated at $37^{\circ} \mathrm{C}$ while shaking at $300 \mathrm{rpm}$. Starting at 4 hours a wet mount was made every hour to count the number of germlings to conidia. Samples were vortexed with $1.0 \mathrm{~mm}$ beads to break up clumps before mounting and fungal germination was quantified manually using the $40 \times$ objective lens of an upright VWR microscope. A minimum of 100 conidia and germlings for each sample were counted.

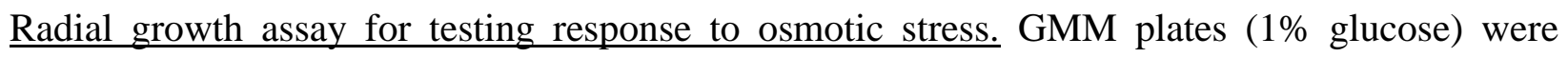
supplemented with $1 \mathrm{M} \mathrm{NaCl}, 1 \mathrm{M} \mathrm{KCl}, 0.5 \mathrm{M} \mathrm{CaCl}_{2}$, or $1 \mathrm{M}$ Sorbitol. $1 \times 10^{5}$ conidia were inoculated onto plates in $2 \mu \mathrm{l}$ drops. Plates were incubated at $37^{\circ} \mathrm{C}$ for 72 hours, at which point the diameter of the colony was measured. Inhibition was determined by normalizing each strain to their 72 hour GMM growth.

Fungal RNA extraction and qRT-PCR analysis for SakA-dependent transcripts. For transcript analysis during growth during osmotic stress, 1x106 conidia of AF293, AF293 $\Delta s a k A$, and LHEVOL were grown in a 6-well plate in $5 \mathrm{~mL}$ of GMM media for 30 hours at $37^{\circ} \mathrm{C}$. Mycelia were transferred to a new 6-well plate with either GMM or GMM supplemented with $1 \mathrm{M} \mathrm{NaCl}$ under sterile conditions and incubated for 30 minutes at $37^{\circ} \mathrm{C}$. Mycelia were transferred to $\mathrm{ZR}$ BashingBead Lysis Tubes ( $0.1 \& 0.5 \mathrm{~mm}$, Zymo Research) and homogenized at $4^{\circ} \mathrm{C}$ using a bead beater for 4 minutes at 250 RPM. RNA was purified from lysate following the manufacturer's protocol for Quick-RNA Fungal/Bacterial Miniprep Kit (Zymo Research). cDNA was synthesized following the manufacturer's protocol for QuantiTect Reverse Transcription Kit (Qiagen) including gDNA removal. Quantitative PCR was performed using SsoAdvanced Universal 
SYBER Green Supermix according to manufacturer's directions and primers described in Supplemental Table 3 using the thermocycler Bio-Rad C-1000 CFX96. Cycling parameters were according to SsoAdvanced Universal SYBER Green Supermix manufacturer recommendation using $60^{\circ} \mathrm{C}$ as the annealing temperature. Transcripts were normalized to actA using the $2^{-\Delta \Delta \mathrm{Ct}}$ method $^{39}$.

Protein Sequence Analysis. Comparison of proteins of the SakA signaling network between CEA10 and Af293 was done using the web Protein BLAST (BLASTp) platform from the US National Library of Medicine, National Center for Biotechnology Information (https://blast.ncbi.nlm.nih.gov/Blast.cgi) using the non-redundant protein sequence database. Protein sequences used for this analysis are listed in Supplemental Table 4.

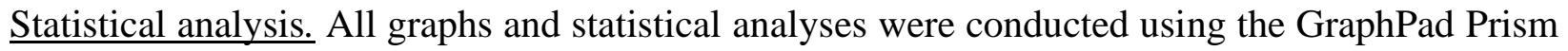
9 software. Statistical significance between experimental groups was determined using Student's t-test (comparison of two experimental groups normally distributed), a Mann-Whitney U test (comparison of two experimental groups that are not normally distributed), a one-way ANOVA using Dunn's post-test (comparison of more than two experimental groups), or two-way ANOVA using Tukey's post-test (comparison of more than two experimental groups with two variables) or Kruskal-Wallis post-test for non-gaussian distributions. 


\section{RESULTS}

In vitro serial passaging of AF293 in lung homogenate medium leads to a strain with increased airway growth.

Previously, we had established that strains of Aspergillus fumigatus, in the context of the lung environment, can be grouped into fast and slow germinators who induce markedly different pathologies ${ }^{4,40}$. Notably, fast germinating strains, such as CEA10, induce extensive loss of alveoli and destruction of the parenchyma with substantial hemorrhaging when compared to slow germinating strains, such as AF293. To identify the potential molecular pathways responsible for the quick germination phenotype within the lung environment by A. fumigatus we utilized an experimental serial passage approach, which enables us to identify potential fungal regulators of growth in the lung microenvironment without knowing the exact nutrients that are biologically available. Specifically, the slow germinating AF293 strain was serially cultured in lung homogenate medium for $8 \mathrm{~h}$ to initiate germination then plated on 1\% GMM plates for 3 days before collecting spores for cataloging and the next passage (Figure 1A). After 13 passages we identified a novel AF293-based strain, referred to as LH-EVOL, that was able to germinate rapidly in lung homogenate medium (Figure 1B). The germination kinetics of the LH-EVOL strain in lung homogenate medium was significantly faster than its parental AF293 strain, while having similar germination kinetics to the CEA10 strain in this in vitro assay (Figure 1B). To determine if the LH-EVOL strain also has an increased germination rate during initial airway challenge of mice,

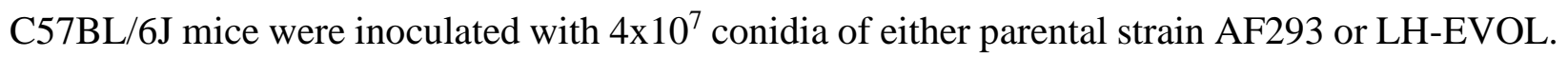
After challenge, significantly more germination by LH-EVOL within the BALF 12 hours post inoculation was observed compared to its parental AF293 strain (Figure 1C). Thus, our in vitro 
serial passaging experimental conditions drove the development of a novel AF293-based strain that can rapidly germinate within the lung airways of mice.

Comparison of pulmonary inflammatory response induced following AF293 and LH-EVOL challenge in the murine lung.

Given the different germination rates of the AF293 and LH-EVOL strains in vivo, we next analyzed the inflammatory response induced in the lung after challenge with each respective strain. Total RNA was extracted from whole lungs of C57BL/6J mice challenged 40 hours prior with either AF293 or LH-EVOL. Changes in mRNA levels of immune-related genes were analyzed using NanoString nCounter technology. We observed strikingly different overall changes in mRNA levels in PBS-challenged animals compared to the two fungal strains (Supplemental Dataset 1-2). Importantly, mRNA levels of 57 genes were significantly increased greater than 2fold following LH-EVOL challenge when compared with AF293 challenge, whereas 65 genes were significantly increased greater than 2-fold following AF293 challenge when compared with LH-EVOL challenge (Figure 2A and Supplemental Dataset 3). When we examined differential gene expression and pathway analysis using nSolver Advanced Analysis software, we noted a dramatic signature related to IFN signaling following AF293 challenge (Figure 2B). This increased IFN signaling signature is evidenced by significant increases in Cxcl9, Cxcl10, Stat1, Zbpl, Irf7, Isg15, and Ifit3 levels (Table 1). It has been established that early anti-fungal immune responses to conidia will induce expression of IFN- $\beta$ and CXCL10 $0^{41}$, therefore increased IFN signaling may occur in AF293 due to slower germination. In contrast, there were dramatic increases across several pathways associated with innate immunity and cytokine/chemokine signaling following LH-EVOL challenge relative to AF293 challenge (Figure 2B). Increased mRNA expression levels 
of pro-inflammatory cytokines including Illa, Il6, Csf2, Cxcl1, Cxcl2, and Cxcl5 and expression levels of the transcription factors $N f k b l$ and Hifla were elevated following LH-EVOL challenge (Table 2).

The marked elevation of Illa transcripts observed following LH-EVOL challenge fits with our previous observation that IL- $1 \alpha$ is essential for host resistance against highly virulent isolates of $A$. fumigatus that can rapidly germinate in the lung airways ${ }^{4,42}$. To test the importance of IL-1 $\alpha$ in host resistance against the LH-EVOL strain, C57BL/6J mice and $I l 1 a^{(-/-)}$mice were challenged with either AF293 or LH-EVOL. At 40 hours post-challenge, lungs were collected for histological analysis to determine fungal germination. We found that not only did C57BL/6J and IL-1 $\alpha$ deficient mice challenged with LH-EVOL have significantly greater fungal germination than AF293 challenged mice (Figure 2C), but IL-1 $\alpha$-deficient mice challenged with LH-EVOL also had significantly more fungal germination than C57BL/6J mice challenged with LH-EVOL (Figure 2C). Taken together these data reveal that the rapid germinating LH-EVOL strain is markedly more inflammatory.

Genomic analysis of LH-EVOL identifies a potential truncation mutation within Afu5g08390 $\underline{(s s k A) .}$

Selective serial culturing of an organism will often result in genetic modifications as the organism responds to a given environmental pressure, such as growth in the lung microenvironment without direct a priori knowledge about the exact nutrients that are biologically available for fungal growth. Therefore, we asked what genomic changes arose in LH-EVOL to determine the genetic basis for its rapid germination phenotype. Three single spore clones of LHEVOL were selected for further analysis. Each of the clones of LH-EVOL were able to germinate 
substantially faster than the parental AF293 strain in LH medium in the in vitro germination assay (Figure 3A). Next, whole genome sequencing of these three clones of LH-EVOL was conducted and compared with its parental AF293 strain. Whole genome sequencing revealed conserved INDEL mutations in Afu5g08390 across the three LH-EVOL clones (Figure 3B). Afu5g08390 encodes for SskA which is a response regulator in the high osmolarity glycerol (HOG) pathway ${ }^{43}$. These INDEL mutations are predicted to insert premature stop codons in the Afu5g08390 gene that may induce premature truncation before the recognition (REC) domain (Figure 3C), a domain necessary for the signaling function of Ssk1, the SskA homologue, in yeast ${ }^{29}$.

\section{$\underline{\text { LH-EVOL has a decreased SakA-dependent response under osmotic stress. }}$}

SskA is a response regulator within the high osmolarity glycerol pathway which drives the activation of the MAPKKK SskB, MAPKK PbsB, and MAPK SakA ${ }^{44,45}$. SakA is critical for maintaining homeostasis in A. fumigatus during osmotic stress ${ }^{13,46,47}$. Based on the predicted truncation of SskA prior to its REC domain (Figure 3C), we hypothesized that the LH-EVOL strain has a decreased SakA signaling response. To directly test this AF293, AF293 $\Delta s a k A$, and LHEVOL were inoculated onto GMM plates and GMM plates supplemented with $1 \mathrm{M} \mathrm{NaCl}, 1 \mathrm{M} \mathrm{KCl}$, $0.5 \mathrm{M} \mathrm{CaCl}_{2}$, and $1 \mathrm{M}$ Sorbitol for 72 hours at $37^{\circ} \mathrm{C}$ (Figure 4A). LH-EVOL grown on GMM formed significantly larger colonies than AF293, similar to AF293 $\Delta$ sakA (Figure 4B). Importantly, when LH-EVOL was grown on GMM plates supplemented with osmotic stressors, its growth was substantially altered compared with AF293, similar to the response of the AF293 $\Delta s a k A$ mutant (Figure 4C) ${ }^{13}$. Specifically, both LH-EVOL and AF293 $\Delta s a k A$ were significantly impaired in their ability to grow in the presence of $1 \mathrm{M} \mathrm{NaCl}$, while growing significantly better in the presence of $1 \mathrm{M}$ sorbitol. 
Since LH-EVOL appears to have an altered response to osmotic stress similar to AF293 $\Delta s a k A$, we next explored the transcriptional responses of AF293, AF293 $\Delta s a k A$, and LH-EVOL conidia to $1 \mathrm{M} \mathrm{NaCl}$, which is known to drive SakA activation and signaling ${ }^{13}$. Each strain was grown in GMM media for 30 hours at $37^{\circ} \mathrm{C}$ before being transferred to either GMM or GMM supplemented with $1 \mathrm{M} \mathrm{NaCl}$ for 30 minutes. We choose to examine sakA expression itself, as well as SakA-dependent transcripts $p t p A, g p d A$, and $p b s B$ which are highly upregulated in AF293 but not in AF293 $\Delta s a k A$ following osmotic stress with $1 \mathrm{M} \mathrm{NaCl}^{13,48}$. Transcript levels of sakA, ptpA, $g p d A$, and $p b s B$ were all increased to a significantly greater extent in parental strain AF293 as opposed to LH-EVOL and AF293 $\triangle s a k A$ when exposed to $1 \mathrm{M} \mathrm{NaCl}$ (Figure 4D). These data support the hypothesis that the allele of Afu5g08390 (sskA) found in the LH-EVOL strain likely encodes a SskA truncation mutant with decrease signal transduction.

Loss of Afu $5 \mathrm{~g} 08390$ (sskA) in AF293 results in increased germination.

Since the INDEL mutations found within Afu5g08390 (sskA) in the LH-EVOL strain are predicted to result in a premature truncation mutation (Figure 3C), which resulted in decreased SakA-dependent transcriptional response to osmotic stress (Figure 4), we next tested whether the complete loss of SskA results in increased germination potential in AF293. To do this, we constructed a sskA null mutant using homologous recombination in the AF293 strain of $A$. fumigatus, as well as an ectopic complementation strain (Supplemental Figure 1). When cultured on GMM plates the AF293 $\Delta s s k A$ strain had significantly greater radial growth than the parental AF293, which was similar to what was observed with the AF293 $\Delta s a k A$ and LH-EVOL strains (Supplemental Figure 2A). Next, to determine if the AF293 $\Delta s s k A$ strain had decreased SakA signaling we explored the transcriptional responses of AF293, AF293 $\Delta s a k A$, and AF293 $\Delta s s k A$ 
biofilms to $1 \mathrm{M} \mathrm{NaCl}$. Each strain was cultured in $\mathrm{GMM}$ media for 30 hours at $37^{\circ} \mathrm{C}$ before being transferred to either GMM or GMM supplemented with $1 \mathrm{M} \mathrm{NaCl}$ for 30 minutes. Similar to previous results (Figure 4D), transcript levels of $s a k A, p t p A, g p d A$, and $p b s B$ were all increased in AF293 relative to AF293 $\Delta s a k A$ when exposed to $1 \mathrm{M} \mathrm{NaCl}$ (Supplemental Figure 2B-E). Importantly, AF293 $\Delta s s k$ failed to show elevated transcript levels of $s a k A, p t p A, g p d A$, and $p b s B$, which supports the hypothesis that SskA-SakA signaling is decreased in the AF293 $\Delta s s k A$ strains (Supplemental Figure 2B-E).

Next, to determine if AF293 $\Delta s s k A$ was able to germinate faster than AF293 or the complemented AF293 $\Delta s s k A^{R C}$ strain, we conducted an in vitro germination assay in lung homogenate medium. When AF293 $\Delta s s k A$ was grown in lung homogenate media, robust germination was observed compared to the parental strain or the complemented AF293 $\Delta s s k A^{R C}$ (Figure 5A). In contrast, all three strains germinated at equivalent rates in nutrient rich GMM + YE medium (Figure 5B), demonstrating a lack of intrinsic changes to the overall germination potential of the AF293 $\Delta s s k A$ strain. To determine if AF293 $\Delta s s k A$ also has an increased germination rate during initial airway infection of mice, C57BL/6J mice were inoculated intratracheally with either AF293, AF293 $\Delta s s k A$, and AF293 $\Delta s s A^{R C}$. Twelve hours post inoculation, AF293 $\Delta s s k A$ had germinated to a significantly greater degree than either the AF293 or AF293 $\Delta s s k A^{R C}$ (Figure 5C). Thus, loss of SskA signaling in AF293 strain of A. fumigatus results in enhanced germination potential within the lung microenvironment.

Loss of the MAPKs $S a k A$ and $M p k C$ results in increased germination.

SakA associates with MpkC, a MAPK of the cell wall integrity pathway, in the nucleus ${ }^{46,49}$. Therefore, we wanted to test the role of both the SakA and MpkC MAPK proteins in the regulation 
of germination of AF293 in the pulmonary environment. To determine if SakA and MpkC regulate A. fumigatus germination, the rate of conidial germination of AF293 $\Delta s a k A$ and AF293 $\Delta m p k C$ mutant strains were assessed in lung homogenate medium. We found that germination indeed increased in both AF293 $\triangle s a k A$ and AF293 $\triangle m p k C$ relative to the parental strain (Figure 6A). Additionally, the germination rate of both AF293 $\Delta s a k A$ and AF293 $\Delta m p k C$ were similar to what we had observed for LH-EVOL and AF293 $\Delta s s k A$ (Figure 1B and Figure 4A, respectively). In contrast, all strains have similar germination rates in GMM + YE medium, demonstrating that there were no global increases to the overall germination potential of these mutants (Figure 6B). To determine if AF293 $\Delta s a k A$ and AF293 $\Delta m p k C$ also have an increased germination rate during initial airway infection of mice, C57BL/6J mice were inoculated intratracheally with either AF293, AF293 $\Delta s a k A$, or AF293 $\Delta m p k C$. Twelve hours post inoculation, both AF293 $\Delta s a k A$ and AF293 $\triangle m p k C$ had germinated to a significantly greater degree than their parental strain (Figure 6C). Thus, SskA signaling appears to converge on both the SakA and MpkC MAPKs in the AF293 strain of A. fumigatus to regulate germination within the lung microenvironment.

Protein sequence analysis of the SakA signaling components in CEA10 identifies differences in $\underline{\text { TcsB and MpkC. }}$

As the CEA10 strain of $A$. fumigatus is able to rapidly germinate both in vitro in lung homogenate medium and in vivo in murine lungs ${ }^{4}$, we explored CEA10's transcriptional response to $1 \mathrm{M} \mathrm{NaCl}$ in the biofilm transfer assay. Specifically, CEA10 was cultured in GMM for 30 hours at $37^{\circ} \mathrm{C}$ before being transferred to either GMM or GMM supplemented with $1 \mathrm{M} \mathrm{NaCl}$ for 30 minutes. While AF293 had elevated transcript levels of $s a k A, p t p A$, gpdA, and $p b s B$, CEA10 did 
not have increased levels of these transcript (Supplemental Figure 2). These data support the hypothesis that SskA-SakA signaling is altered in CEA10 relative to AF293.

Next, we compared the SskA amino acid sequences between AF293 and CEA10. Using BLASTp we found that the SskA protein sequences where 100\% identical between AF293 and CEA10 (Supplemental Table 4). Next, we expanded our BLASTp analysis to include all the known components of the SskA-SakA signaling pathway. This analysis revealed that YpdA, SskB, PbsB, and SakA were also 100\% identical between Af293 and CEA10 (Supplemental Figure 2). In contrast, BLASTp sequence alignment of the putative histidine kinase response regulator TcsB, homologue to $S$. cerevisiae SLN1 and integral to the activation of Ssk1 in yeast ${ }^{44,50,51}$, revealed a 13 amino acid deletion (AA 709-721, XP_001481640.1) within the predicted ATPase (AA 696863, XP_001481640.1) domain in the histidine kinase domain of the CEA10 TcsB protein that is not present in AF293 (Supplemental Figure 2). Additionally, BLASTp sequence alignment of MpkC, which can interact with SakA signaling $46,49,52,53$, shows 4 non-synonymous mutations (Supplemental Table 4). Thus, our BLASTp analysis suggest that changes in the SskA protein of CEA10 are likely not responsible for the increased germination rate of that strain, while change in other proteins in the SakA signaling network, particularly TcsB, might be functionally different.

$\underline{\text { SskA-SakA signaling limits A. fumigatus germination in decreased glucose conditions. }}$

The ASL which lines the airway epithelium including the lungs is a key component of respiratory homeostasis and facilitates mucociliary clearance of foreign particulates and pathogens. The glucose concentration of ASL is approximately $0.4 \mathrm{~m} M$, substantially lower than typical in vitro culturing conditions. In yeast, glucose starvation can mediate activation of the stress MAPK Hog1 in a Ssk1-dependent manner ${ }^{53}$. Consequently, we hypothesized that limited glucose 
availability may limit AF293 germination through SskA-SakA activation. To test this hypothesis, an in vitro germination assay using the AF293, LH-EVOL, AF293 $\Delta s s k$, AF293 $\Delta s a k A$, and AF293 $\triangle m p k C$, strains was conducted in either 1\% GMM (55.56 mM of glucose) or 0.25\% GMM (13.89 $\mathrm{mM}$ of glucose). Interestingly, we found that while AF293 underwent limited germination in $1 \%$ GMM it nearly completely failed to germinate in $0.25 \%$ GMM (Figure 7A); however, the AF293 $\Delta s s k A$ (Figure 7C), AF293 $\Delta s a k A$ (Figure 7D), and AF293 $\Delta m p k C$ (Figure 7E) underwent greater germination in $0.25 \%$ GMM than in $1 \%$ GMM. When we examined LH-EVOL we found that the germination rate in both $1 \%$ GMM and $0.25 \%$ GMM were equivalent (Figure 5B). Thus, it appears that in the absence of SskA-SakA signaling, there is an increased potential for $A$. fumigatus strain to germinate under low glucose conditions which would be found in the airways upon initial deposition. 


\section{DISCUSSION}

After entering the airways of a mammalian host, conidia are typically cleared by mucociliary clearance or innate phagocytotic responses ${ }^{1-3,54}$. In order to drive disease within a host, two types of virulence traits have been proposed: (1) disease initiating factors, which are factors that either promote the initiation of infection or disease pathology; and (2) disease progressing factors, which facilitate microbe persistence, host damage, and disease progression in an established infection microenvironment ${ }^{55}$. Our previous work has demonstrated early fungal germination within the airway space is a disease initiating trait in a model of $A$. fumigatus induced bronchopneumonia ${ }^{4}$. However, the molecular mechanism(s) responsible for driving the increased fungal germination rate of certain A. fumigatus isolates remains elusive. In this study, we took an experimental serial passage approach to identify factors that may drive enhanced fungal germination. Through this approach we isolated a novel, lung-adapted strain, LH-EVOL, from the parental AF293 strain. The LH-EVOL strain showed increased germination in vivo in murine airways and in vitro in lung homogenate medium (Figure 1) in addition to enhanced proinflammatory cytokine responses (Figure 2).

Whole genome sequencing of LH-EVOL revealed the presence of INDEL mutations in SskA that are predicted to result in the truncation of the SskA recognition (REC) domain ${ }^{37}$ (Figure 3), a domain necessary for SskA to dimerize with SskB to phosphorylate PbsB. In support of a predicted truncation of SskA's REC domain, the LH-EVOL strain does not induce as strong of a SakA-dependent response as measured by transcript abundance of SakA target genes during osmotic stress (Figure 4); thus, the sskA allele identified in the LH-EVOL strain likely encodes an altered or non-functional SskA protein. However, one limitation of this study is that the function of the SskA protein found in LH-EVOL has not been directly determined. The prediction that the 
LH-EVOL allele of $s s k A$ result in a non-functional protein is, however, further supported by the finding that AF293 $\Delta s s k A$ also germinates more rapidly than the parental strain in vitro in lung homogenate medium (Figure 5A) and in vivo in the murine airways (Figure 5C) which is what was observed in the LH-EVOL strain (Figure 1). SskA is part of the SakA MAPK stress response pathway and has been shown to be important for SakA activation in the Afs35 strain of $A$. fumigatus $^{26}$. During germination of A. nidulans, SakA is dephosphorylated and exits the nucleus ${ }^{49}$. Based on the location of the predicted premature stop codon due to an INDEL mutation found in the sskA gene of LH-EVOL (Figure 3), it can be predicted that there would be less phosphorylated SakA in LH-EVOL and AF293 $\Delta s s k$, making those strains poised to germinate quickly within the lung airways. This is further supported by our data demonstrating that AF293 $\Delta s a k A$ also exhibits increased germination rates in vitro in lung homogenate medium (Figure 6A) and in vivo in the airways (Figure 6C). Interestingly, AF293 $\Delta s a k A$ appears to undergo greater germination potential in the murine lungs than AF293 $\Delta s s k A$, although this was not compared directly. In $S$. cerevisiae Hog1 signaling has two branches for its activation: Sln1 and Sho1 which are Ssk1dependent and Ssk1-independent, respectively ${ }^{56}$, which also seems to be case in A. fumigatus $^{57}$. Our study only examined the role of the SskA-dependent branch in A. fumigatus germination, but future studies should examine the interplay of these two SakA signaling branches in regulating $A$. fumigatus germination.

As CEA10 is also a strain of $A$. fumigatus that can rapidly germinate in the lungs ${ }^{4}$, we next examined the SskA-SakA signaling network in that strain. Interestingly, compared with AF293, CEA10 had a significantly weaker induction of SakA-dependent transcripts (Supplemental Figure 2). However, the amino acid sequence identity of SskA and SakA where $100 \%$ between AF293 and CEA10. Upstream of SskA the histidine kinase TcsB, a homologue of SLN1 in yeast that is 
necessary for Ssk1 and HOG1 activation ${ }^{44,50,51}$, in CEA10 has a 13 amino acid deletion in the predicted ATPase domain (Supplemental Figure 3). This deletion could account for reduced expression of $p b s B, s a k A$ and SakA dependent genes in CEA10 during osmotic stress (Supplemental Figure 2), but this needs to be experimentally further explored. Therefore, strains of A. fumigatus with decreased activity of SakA appear to have an increased potential for rapid germination and growth in murine lungs. Future studies should look at the relationship of $A$. fumigatus germination and the activity of specific SakA-dependent genes.

Within the pulmonary environment, the specific nutrient(s) driving A. fumigatus germination remains elusive. The airways of the lungs are a low-nutrient environment. Recent in vivo transcriptional analysis of the response of A. fumigatus in the murine airways demonstrates that $A$. fumigatus undergoes significant stress from iron, zinc, and nitrogen starvation ${ }^{58}$. In our experiments both the LH-EVOL strain and AF293-based SskA null mutant have an increased ability to germinate in 1\% GMM when compared to their parental AF293 strain (Figure 5). Furthermore, the AF293-based SakA-null mutant has an increased ability to germinate in $1 \%$ $\mathrm{GMM}^{13}$. Interestingly, the ability of wild-type AF293 to germinate can be rescued by the supplementation of GMM with yeast extract (Figure 5B) or complete medium containing yeast extract, peptone, and tryptone ${ }^{13}$. This is significant because it has previously been shown that AF293 conidia are unable to utilize nitrate, the nitrogen source available in GMM media, as effectively as AF293 $\Delta s a k A$ for growth in a SakA-dependent manner ${ }^{13}$. Taken together these data suggest that $A$. fumigatus may need to be able to effectively utilize non-preferred nitrogen sources within the lung microenvironment to initiate germination, which will be explored in future studies.

Glucose levels in ASL of a healthy adult are approximately $0.4 \mathrm{~m} M^{14}$. These levels are $\sim 10$-fold reduced when compared with plasma ${ }^{14}$ and is thought to limit microbial growth thus 
limiting infections ${ }^{15}$. Surprisingly, we found that the LH-EVOL and AF293-based SskA, SakA, and $\mathrm{MpkC}$ null mutants were still able to germinate in $0.25 \%$ GMM, whereas the parental AF293 isolate was not. SakA has previously been shown to be directly involved in glucose uptake, glycogen and trehalose storage, and trehalose utilization by A $_{\text {fumigatus }}^{59}$. In addition, SakAdependent glucose sensing modulates biofilm development ${ }^{60}$. Furthermore, in Cryptococcus neoformans, Hog1 and protein kinase A (PKA) have been shown to be critical for adaptation to the glucose-limited environment of the lungs and glucose-replete environment of the brain, respectively ${ }^{61}$. In low glucose conditions, $\operatorname{Hog} 1$ of $C$. neoformans becomes activated, resulting in translational repression through decreased ribosome biogenesis which is necessary for stress adaptation to the low glucose environment of the lungs. In A. fumigatus, PKA signaling, regulated by SakA and $\mathrm{MpkC}^{59}$, has been shown to be critical for growth in low glucose environments and conidial germination ${ }^{62}$. Considering these findings, our data would predict that the low glucose environment of the lungs might similarly drive SakA activation in some A. fumigatus strains, such as AF293, limiting germination but increasing resistance against physiological stressors. In contrast, rapidly germinating strains may be more susceptible to osmotic and oxidative stressors in the host environment due to decreased SakA activity. This fits with the observation that CEA10 is more rapidly cleared than AF293 in a zebrafish model of infection ${ }^{5}$. Consequently, SakA could be particularly important for A. fumigatus to adapt to the cystic fibrosis (CF) lung environment where there is increased oxidative stress ${ }^{63}$ and ASL glucose concentrations can be 10 times higher than healthy adults ${ }^{16}$. Ross et al (2021) demonstrated a distinct relationship between activation of the SakA pathway and adaptation by A. fumigatus to the lung environment in a CF patient ${ }^{48}$. In that study, PbsB, an intermediary kinase between SskA and SakA, was found to have a missense mutation that resulted in increased SakA activity in response to osmotic stress $^{48}$. These data 
suggest a potentially important and distinct relationship between SakA activity in A. fumigatus in response to nutritional and osmotic stressors in the pulmonary environment, which may be critical to understanding disease initiation and progression within both acute and chronic models of aspergillosis.

Finally, our inflammatory profiling of pulmonary responses to both AF293 and LH-EVOL reinforces our knowledge that early germination in lung airways is a key driver of the distinct immunopathological phenotypes observed within different A. fumigatus isolates, where fast germinating isolates require an IL-1 $\alpha$ dependent host response to maintain host resistance ${ }^{4}$ (Figure 2C). Our inflammatory profiling also found that Hifla mRNA was expressed to a greater extent following LH-EVOL challenge than AF293 (Table 2), fitting with our previous observation that HIF $1 \alpha$-dependent inflammatory responses are necessary for host resistance against the rapidly germinating CEA10 strain of A. fumigatus, but not $\mathrm{AF} 293^{40}$.

Notably, we found a stronger IFN signaling signature following challenge with AF293 than LH-EVOL (Figure 2B). Recent work has demonstrated a critical role for both type I and type III IFNs in driving host resistance against A. $_{\text {fumigatus }}^{64}$. Based on our NanoString analysis (Figure 2), slow germinating strains of A. fumigatus may be more potent inducers of the type I and/or type III IFN response, which warrants further exploration. In support of this, human bronchial epithelial cells respond to resting conidia, rather than swollen conidia or hyphae, by enhanced secretion of IFN- $\beta$ and CXCL10 ${ }^{41}$. The expression of type I IFN (IFN- $\alpha$ and IFN- $\beta$ ) and CXCL10 can be induced by $A$. fumigatus dsRNA through both TLR3-Trif and MDA5-MAVS dependent signaling ${ }^{41,65,66}$. Overall, this suggests that the induction of IFN may occur in response to early development stages of $A$. fumigatus infection, while the robust pro-inflammatory response (e.g. IL-1 $\alpha$ release) is driven by the later invasive hyphal stage. Differential host response to other 
fungal infections over time have been previously described. Recent work examining the inflammatory response of vaginal epithelial cells to Candida spp. demonstrate an early, homogeneous type I IFN response to all Candida spp., but at later time points, the inflammatory response diverges in a species specific and damage-dependent manner ${ }^{67}$.

In conclusion, this study further emphasizes the strain-specific virulence, pathology, and inflammatory responses that occur during A. fumigatus infections. Our study also identifies a role for the loss of SakA MAPK signaling in enhancing conidial germination, particularly in low glucose environments. This likely comes with the recompense of decreased resistance to osmotic and oxidative stress, which could be critical to disease progression during chronic fungal diseases ${ }^{67}$. Thus, future studies must address the role of these traits and pathways in both acute and chronic models of aspergillosis. 


\section{AUTHORS CONTRIBUTIONS}

Conceived the experiments: MEK, RAC, JJO. Designed the experiments: MEK, RMT, AKC, CHK, BSR, RAC, JJO. Performed the experiments: MEK, MS, RMT, AKC, BSR. Generated fungal mutants: CHK, DM. Analyzed the data: MEK, MS, AKC, CHK, DM, LAL, JES, RAC, JJO. Wrote the paper: MEK, JJO.

\section{ACKNOWLEDGEMENTS}

Thank you to Drs. Deborah Hogan and James Bliska (Geisel School of Medicine at Dartmouth) for helpful discussion on this project and manuscript. The authors thank Dr. Charles Puerner (Dartmouth College, Cramer Lab) for quantifying the glucose levels in our lung homogenate medium. The authors thank Dr. Jong Heon Kim (USDA ARS, Albany, CA) for providing the AF293 $\triangle s a k A$ and AF293 $\Delta m p k C$ strains. Research in this study was supported in part by institutional startup funds to JJO in part through the Dartmouth Lung Biology Center for Molecular, Cellular, and Translational Research grant P30 GM106394 and Center for Molecular, Cellular and Translational Immunological Research grant P30 GM103415. JJO was partially supported by NIH R01 AI139133 grant. MEK and RMT were supported by Immunology Training Program (NIH/NIAID T32 AI007363). BSR was supported by the Dartmouth Cystic Fibrosis Training Program (NIH/NHLBI T32 HL134598). RAC, LAL, and JES were partially supported by NIH R01 AI130128. JS is a CIFAR Fellow in the Fungal Kingdom: Threats and Opportunities program. CHK was partially supported by NIH F31 AI138354. The funders had no role in the preparation or publication of the manuscript. 


\section{REFERENCES}

1. Latge, J.-P. Aspergillus fumigatus and Aspergillosis. CLIN MICROBIOL REV 12, 41 (1999).

2. van de Veerdonk, F. L., Gresnigt, M. S., Romani, L., Netea, M. G. \& Latgé, J.-P. Aspergillus fumigatus morphology and dynamic host interactions. Nat. Rev. Microbiol. 15, 661-674 (2017).

3. Latgé, J.-P. \& Chamilos, G. Aspergillus fumigatus and Aspergillosis in 2019. Clin. Microbiol. Rev. 33, 75 (2020).

4. Caffrey-Carr, A. K. et al. Interleukin $1 \alpha$ Is Critical for Resistance against Highly Virulent Aspergillus fumigatus Isolates. Infect. Immun. 85, e00661-17, e00661-17 (2017).

5. Rosowski, E. E. et al. Macrophages inhibit Aspergillus fumigatus germination and neutrophil-mediated fungal killing. PLOS Pathog. 14, 28 (2018).

6. Rizzetto, L. et al. Strain Dependent Variation of Immune Responses to A. fumigatus: Definition of Pathogenic Species. PLoS ONE 8, e56651 (2013).

7. Osherov, N. \& May, G. Conidial Germination in Aspergillus nidulans Requires RAS Signaling and Protein Synthesis. Genetics 155, 647-656 (2000).

8. Shin, K.-S., Kwon, N.-J. \& Yu, J.-H. GßY-mediated growth and developmental control in Aspergillus fumigatus. Curr. Genet. 55, 631-641 (2009).

9. Lafon, A., Seo, J.-A., Han, K.-H., Yu, J.-H. \& d’Enfert, C. The Heterotrimeric G-Protein $\operatorname{GanB}(\alpha)-\operatorname{Sfa}(\beta)-G p g A(\gamma)$ Is a Carbon Source Sensor Involved in Early cAMP-Dependent Germination in Aspergillus nidulans. Genetics 171, 71-80 (2005). 
10. Zhao, W. et al. Deletion of the Regulatory Subunit of Protein Kinase A in Aspergillus fumigatus Alters Morphology, Sensitivity to Oxidative Damage, andVirulence. Infect. Immun. 74, 4865-4874 (2006).

11. Juvvadi, P. R. et al. Localization and activity of the calcineurin catalytic and regulatory subunit complex at the septum is essential for hyphal elongation and proper septation in Aspergillus fumigatus: Analysis of the calcineurin complex in Aspergillus fumigatus. Mol. Microbiol. 82, 1235-1259 (2011).

12. Cramer, R. A. et al. Calcineurin Target CrzA Regulates Conidial Germination, Hyphal Growth, and Pathogenesis of Aspergillus fumigatus. Eukaryot. Cell 7, 1085-1097 (2008).

13. Xue, T., Nguyen, C. K., Romans, A. \& May, G. S. A Mitogen-Activated Protein Kinase That Senses Nitrogen Regulates Conidial Germination and Growth in Aspergillus fumigatus. Eukaryot. Cell 3, 557-560 (2004).

14. Baker, E. H. \& Baines, D. L. Airway Glucose Homeostasis. Chest 153, 8 (2018).

15. Pezzulo, A. A. et al. Glucose Depletion in the Airway Surface Liquid Is Essential for Sterility of the Airways. PLoS ONE 6, 8 (2011).

16. Garnett, J. P., Baker, E. H. \& Baines, D. L. Sweet talk: insights into the nature and importance of glucose transport in lung epithelium. Eur. Respir. J. 40, 1269-1276 (2012).

17. Garnett, J. P. et al. Proinflammatory Mediators Disrupt Glucose Homeostasis in Airway Surface Liquid. J. Immunol. 189, 373-380 (2012).

18. Mallia, P. et al. Role of airway glucose in bacterial infections in patients with chronic obstructive pulmonary disease. J. Allergy Clin. Immunol. 142, 815-823.e6 (2018). 
19. Garnett, J. P. et al. Hyperglycaemia and Pseudomonas aeruginosa acidify cystic fibrosis airway surface liquid by elevating epithelial monocarboxylate transporter 2 dependent lactate-H+ secretion. Sci. Rep. 6, 37955 (2016).

20. Oliveira, T. L. et al. SGLT1 activity in lung alveolar cells of diabetic rats modulates airway surface liquid glucose concentration and bacterial proliferation. Sci. Rep. 6, 21752 (2016).

21. Gill, S. K. et al. Increased airway glucose increases airway bacterial load in hyperglycaemia. Sci. Rep. (2016) doi:10.1038/srep27636.

22. Baddley, J. W. Clinical risk factors for invasive aspergillosis. Med. Mycol. 49, S7-S12 (2011).

23. Düesberg, U., Wosniok, J., Naehrlich, L., Eschenhagen, P. \& Schwarz, C. Risk factors for respiratory Aspergillus fumigatus in German Cystic Fibrosis patients and impact on lung function. Sci. Rep. 10, 18999 (2020).

24. Ghanaat, F. \& Tayek, J. A. Weight loss and diabetes are new risk factor for the development of invasive aspergillosis infection in non-immunocompromized humans. Clin. Pract. (2017) doi:10.4172/clinical-practice.1000125.

25. Knutsen, A. P. Allergic bronchopulmonary aspergillosis in asthma. Expert Rev. Clin. Immunol. 13, 11-14 (2017).

26. Hagiwara, D. et al. NikA/TcsC Histidine Kinase Is Involved in Conidiation, Hyphal Morphology, and Responses to Osmotic Stress and Antifungal Chemicals in Aspergillus fumigatus. PLOS ONE 8, e80881 (2013).

27. Griffiths, L. J. et al. Comparison of DNA extraction methods for Aspergillus fumigatus using real-time PCR. J. Med. Microbiol. 55, 1187-1191 (2006). 
28. Stajich, J. E. et al. FungiDB: an integrated functional genomics database for fungi. Nucleic Acids Res. 40, D675-D681 (2012).

29. Basenko, E. et al. FungiDB: An Integrated Bioinformatic Resource for Fungi and Oomycetes.

$$
\text { J. Fungi 4, } 39 \text { (2018). }
$$

30. Li, H. Toward better understanding of artifacts in variant calling from high-coverage samples. Bioinformatics 30, 2843-2851 (2014).

31. Li, H. et al. The Sequence Alignment/Map format and SAMtools. Bioinformatics 25, 20782079 (2009).

32. Auwera, G. A. et al. From FastQ Data to High-Confidence Variant Calls: The Genome Analysis Toolkit Best Practices Pipeline. Curr. Protoc. Bioinforma. 43, (2013).

33. Poplin, R. et al. Scaling accurate genetic variant discovery to tens of thousands of samples. http://biorxiv.org/lookup/doi/10.1101/201178 (2017) doi:10.1101/201178.

34. Cingolani, P. et al. A program for annotating and predicting the effects of single nucleotide polymorphisms, SnpEff: SNPs in the genome of Drosophila melanogaster strain $\mathrm{w}^{1118}$; iso2; iso-3. Fly (Austin) 6, 80-92 (2012).

35. Horai, R. et al. Production of Mice Deficient in Genes for Interleukin (IL)-1_, IL-1 $1_{L}, I L-1_{-} / N_{L}$, and IL-1 Receptor Antagonist Shows that IL-1 ${ }^{\mathrm{N}}$ Is Crucial in Turpentine-induced Fever Development and Glucocorticoid Secretion. 187, 1463-1475 (1998).

36. Szewczyk, E. et al. Fusion PCR and gene targeting in Aspergillus nidulans. Nature Protocols $1,3111(2006)$.

37. Dhingra, S. et al. RbdB, a Rhomboid Protease Critical for SREBP Activation and Virulence in Aspergillus fumigatus. mSphere 1, mSphere.00035-16, e00035-16 (2016). 
38. Grahl, N. et al. In vivo Hypoxia and a Fungal Alcohol Dehydrogenase Influence the Pathogenesis of Invasive Pulmonary Aspergillosis. PLoS Pathog. 7, e1002145 (2011).

39. Livak, K. J. \& Schmittgen, T. D. Analysis of Relative Gene Expression Data Using Real-Time Quantitative PCR and the 2- $\Delta \Delta C T$ Method. Methods 25, 402-408 (2001).

40. Caffrey-Carr, A. K. et al. Host-Derived Leukotriene B4 Is Critical for Resistance against Invasive Pulmonary Aspergillosis. Front. Immunol. 8, (2018).

41. Beisswenger, C., Hess, C. \& Bals, R. Aspergillus fumigatus conidia induce interferonsignalling in respiratory epithelial cells. Eur. Respir. J. 39, 411-418 (2012).

42. Caffrey, A. K. et al. IL-1 $\alpha$ Signaling Is Critical for Leukocyte Recruitment after Pulmonary Aspergillus fumigatus Challenge. PLOS Pathog. 11, e1004625 (2015).

43. Cheetham, J. et al. A Single MAPKKK Regulates the Hog1 MAPK Pathway in the Pathogenic Fungus Candida albicans $\square$ D. Mol. Biol. Cell 18, 12 (2007).

44. Posas, F. Activation of the yeast SSK2 MAP kinase kinase kinase by the SSK1 two-component response regulator. EMBO J. 17, 1385-1394 (1998).

45. Shivarathri, R. et al. The Two-Component Response Regulator Ssk1 and the MitogenActivated Protein Kinase Hog1 Control Antifungal Drug Resistance and Cell Wall Architecture of Candida auris. mSphere 5, e00973-20, /msphere/5/5/mSphere973-20.atom (2020).

46. Manfiolli, A. O. et al. Aspergillus fumigatus High Osmolarity Glycerol Mitogen Activated Protein Kinases SakA and MpkC Physically Interact During Osmotic and Cell Wall Stresses. Front. Microbiol. 10, 918 (2019). 
47. Bruder Nascimento, A. C. M. de O. et al. Mitogen activated protein kinases SakA ${ }^{\mathrm{HOG}}$ and MpkC collaborate for Aspergillus fumigatus virulence: Mitogen activated protein kinases. Mol. Microbiol. 100, 841-859 (2016).

48. Ross, B. S., Lofgren, L. A., Ashare, A., Stajich, J. E. \& Cramer, R. A. Aspergillus fumigatus InHost HOG Pathway Mutation for Cystic Fibrosis Lung Microenvironment Persistence. mBio 12, 21 (2021).

49. Garrido-Bazán, V., Jaimes-Arroyo, R., Sánchez, O., Lara-Rojas, F. \& Aguirre, J. SakA and MpkC Stress MAPKs Show Opposite and Common Functions During Stress Responses and Development in Aspergillus nidulans. Front. Microbiol. 9, 12 (2018).

50. Posas, F. et al. Yeast HOG1 MAP Kinase Cascade Is Regulated by a Multistep Phosphorelay Mechanism in the SLN1-YPD1-SSK1 "Two-Component" Osmosensor. Cell 86, 865-875 (1996).

51. Dexter, J. P., Xu, P., Gunawardena, J. \& McClean, M. N. Robust network structure of the SIn1-Ypd1-Ssk1 three-component phospho-relay prevents unintended activation of the HOG MAPK pathway in Saccharomyces cerevisiae. BMC Syst. Biol. 9, 17 (2015).

52. Reyes, G., Romans, A., Nguyen, C. K. \& May, G. S. Novel Mitogen-Activated Protein Kinase MpkC of Aspergillus fumigatus Is Required for Utilization of Polyalcohol Sugars. Eukaryot. Cell 5, 1934-1940 (2006).

53. Piao, H. \& Freed, J. M. Metabolic Activation of the HOG MAP Kinase Pathway by Snf1/AMPK Regulates Lipid Signaling at the Golgi. Traffic 13, 1522-31 (2012).

54. Fernandes, J. et al. Penetration of the Human Pulmonary Epithelium by Aspergillus fumigatus Hyphae. J. Infect. Dis. 218, 1306-1313 (2018). 
55. Cramer, R. A. \& Kowalski, C. H. Is It Time To Kill the Survival Curve? A Case for Disease Progression Factors in Microbial Pathogenesis and Host Defense Research. mBio 12, e03483-20, /mbio/12/1/mBio.03483-20.atom (2021).

56. Takayama, T., Yamamoto, K., Saito, H. \& Tatebayashi, K. Interaction between the transmembrane domains of Sho1 and Opy2 enhances the signaling efficiency of the Hog1 MAP kinase cascade in Saccharomyces cerevisiae. PLOS ONE 14, e0211380 (2019).

57. Silva, L. P. et al. Putative Membrane Receptors Contribute to Activation and Efficient Signaling of Mitogen-Activated Protein Kinase Cascades during Adaptation of Aspergillus fumigatus to Different Stressors and Carbon Sources. mSphere 5, (2020).

58. Liu, H. et al. Determining Aspergillus fumigatus transcription factor expression and function during invasion of the mammalian lung. PLOS Pathog. 17, e1009235 (2021).

59. de Assis, L. J. et al. Protein Kinase A and High-Osmolarity Glycerol Response Pathways Cooperatively Control Cell Wall Carbohydrate Mobilization in Aspergillus fumigatus. 9, 15 (2018).

60. Manfiolli, A. O. et al. Mitogen-Activated Protein Kinase Cross-Talk Interaction Modulates the Production of Melanins in Aspergillus fumigatus. mBio 10, e00215-19, /mbio/10/2/mBio.00215-19.atom (2019).

61. Banerjee, D., Bloom, A. L. M. \& Panepinto, J. C. Opposing PKA and Hog1 signals control the post-transcriptional response to glucose availability in Cryptococcus neoformans. Mol. Microbiol. 15 (2016). 
62. Fuller, K. K. et al. Divergent Protein Kinase A isoforms co-ordinately regulate conidial germination, carbohydrate metabolism and virulence in Aspergillus fumigatus: PkaC2 in the growth and virulence of Aspergillus fumigatus. Mol. Microbiol. 79, 1045-1062 (2011).

63. Ciofu, O., Mandsberg, L. F., Wang, H. \& Høiby, N. Phenotypes selected during chronic lung infection in cystic fibrosis patients: implications for the treatment of Pseudomonas aeruginosa biofilm infections. FEMS Immunol. Med. Microbiol. 65, 215-225 (2012).

64. Espinosa, V. et al. Type III interferon is a critical regulator of innate antifungal immunity. Sci. Immunol. 2, eaan5357 (2017).

65. Wang, X. et al. MDA5 Is an Essential Sensor of a Pathogen-Associated Molecular Pattern Associated with Vitality That Is Necessary for Host Resistance against Aspergillus fumigatus. J. Immunol. 205, 3058-3070 (2020).

66. Carvalho, A. et al. TLR3 essentially promotes protective class I-restricted memory CD82 Tcell responses to Aspergillus fumigatus in hematopoietic transplanted patients. 119, 11 (2012).

67. Pekmezovic, M. et al. Candida pathogens induce protective mitochondria-associated type I interferon signalling and a damage-driven response in vaginal epithelial cells. Nat. Microbiol. 6, 643-657 (2021).

68. Nierman, W. C. et al. Genomic sequence of the pathogenic and allergenic filamentous fungus Aspergillus fumigatus. Nature 438, 1151-1156 (2005).

69. Girardin, H., Latgé, J. P., Srikantha, T., Morrow, B. \& Soll, D. R. Development of DNA probes for fingerprinting Aspergillus fumigatus. J. Clin. Microbiol. 31, 1547-1554 (1993). 
Table 1. Select gene transcripts upregulated following AF293 challenge.

\begin{tabular}{ccc} 
GENE & $\begin{array}{c}\text { FOLD CHANGE } \\
\text { (LOG2) }\end{array}$ & BY P-VALUE \\
\hline CAME & 2.92 & 0.0155 \\
Cxcl10 & 2.68 & 0.000138 \\
Gzma & 2.57 & 0.000536 \\
Stat1 & 2.11 & 0.000174 \\
Angpt1 & 2.07 & 0.00212 \\
Nlrc5 & 2.04 & 0.00163 \\
Zbp1 & 1.73 & 0.00243 \\
Ddx60 & 1.66 & 0.00553 \\
Gbp5 & 1.52 & 0.0273 \\
Irf7 & 1.35 & 0.00715 \\
Isg15 & 1.32 & 0.0182 \\
Ifit3 & 1.27 & 0.0357 \\
Ddx58 & 0.664 & 0.0166 \\
Ifih1 & 0.296 & \\
\hline
\end{tabular}


Table 2. Select gene transcripts upregulated following LH-EVOL challenge.

\begin{tabular}{ccc} 
GENE & $\begin{array}{c}\text { FOLD CHANGE } \\
\text { (LOG2) }\end{array}$ & BY P-VALUE \\
\hline NAME & 4.34 & 0.000918 \\
Csf3 & 3.57 & 0.000115 \\
Cxcl2 & 2.59 & 0.000423 \\
Cxcl1 & 2.47 & 0.000918 \\
Il6 & 2.32 & 0.00212 \\
Ptgs 2 & 2.20 & 0.000369 \\
Hifla & 1.51 & 0.000249 \\
Csf2 & 1.51 & 0.000369 \\
Cxcl5 & 1.42 & 0.00515 \\
Ill2a & 1.23 & 0.00442 \\
Nfkbia & 1.08 & 0.000139 \\
Illa & 1.06 & 0.000369 \\
Nfkb1 & 0.77 & 0.000369 \\
Illb & 0.66 & 0.00139 \\
\hline
\end{tabular}


(A)
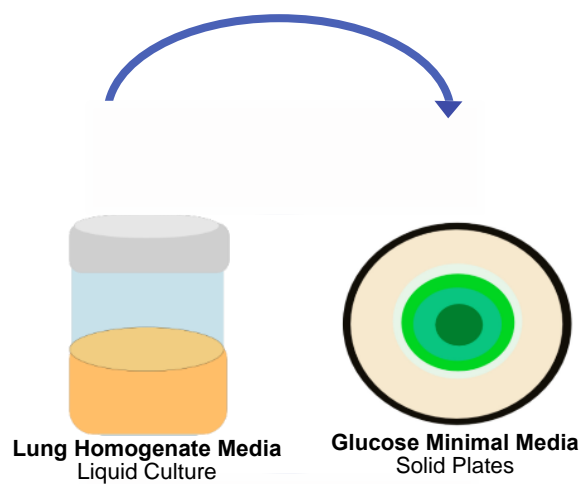

Solid Plates

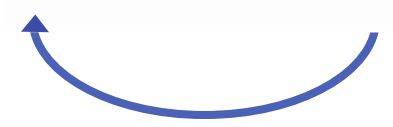

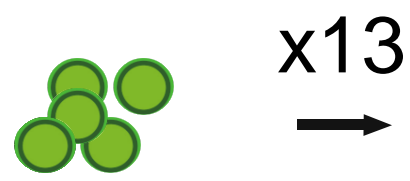

AF293

(B)

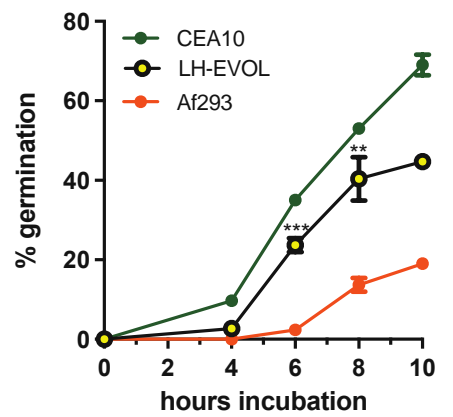

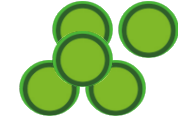

LH-EVOL

(C)

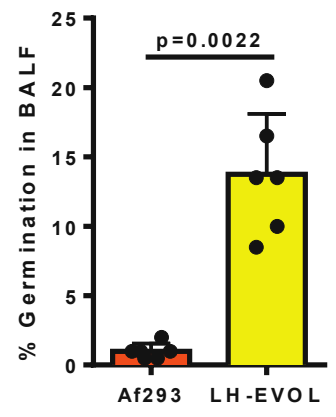

Figure 1. Lung evolved (LH-EVOL) A. fumigatus demonstrates increased in vitro and in vivo germination kinetics. (A) LH-EVOL was generated through serial passaging of the AF293 strain thirteen times in lung homogenate media. (B) Lung tissue from C57BL/6 mice was homogenized in PBS and used as the medium for in vitro germination assays. Percent germination over time in lung homogenate media of AF293; CEA10, or LH-EVOL. was quantified every $2 \mathrm{~h}$ by microscopically counting the numbers of conidia and germlings. Data are represented as the percentage of fungal matter that was germinated. Data are representative of at least 2 independent experiments consisting of 3 biological replicates per group. Each symbol represents the group mean \pm 1 standard error of the mean. (C) C57BL/6J mice were challenged with $4 \times 10^{7}$ conidia of AF293 or LH-EVOL. At $12 \mathrm{hpi}$, mice were euthanized, and BALF was collected to quantify germination in the airways. Data are representative of results from 2 independent experiments with 5 mice per group. (B, C) Statistical significance was assessed between LH-EVOL and AF293 using a Mann-Whitney U-test. $(* *)$ indicates a $p$-value $\leq 0.01 ;(* * *)$ indicates a $p$-value $\leq 0.001$. 
A

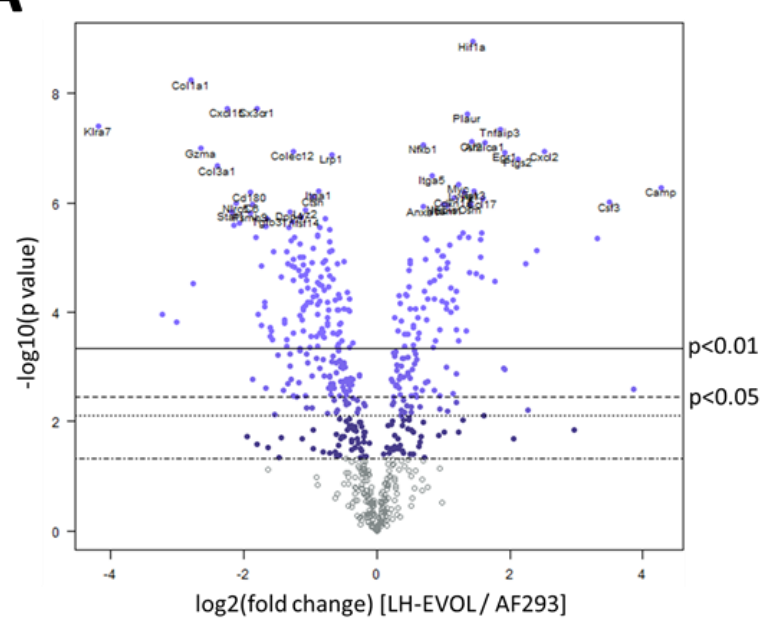

C

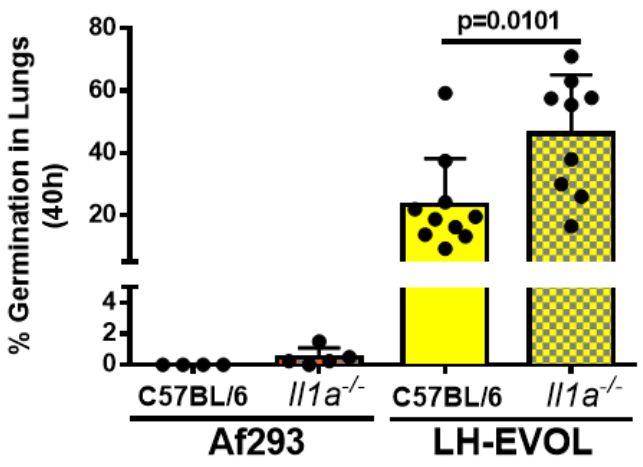

B

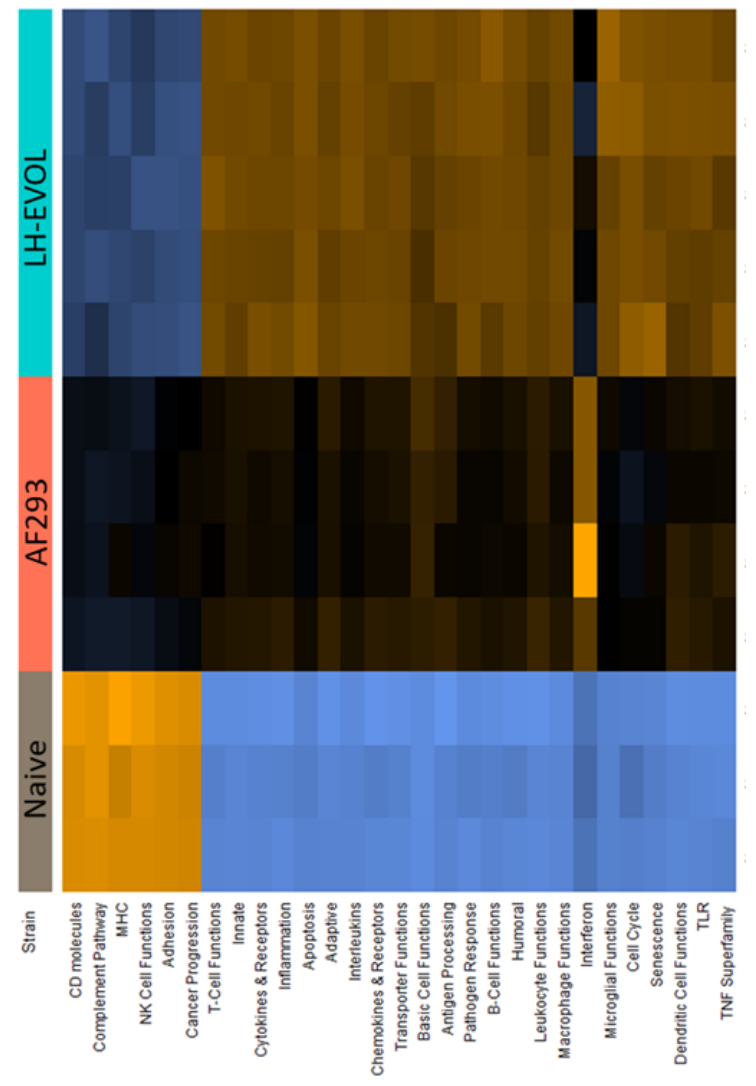

Figure 2. Differential pulmonary inflammatory gene expression and immune response after AF293 and LH-EVOL challenge. C57BL/6 mice were inoculated with either PBS or $4 \times 10^{7}$ AF293 or LH-EVOL conidia and euthanized at $40 \mathrm{~h}$ post-inoculation. Total mRNA was extracted from the whole lung, and gene expression was analyzed by NanoString nCounter PanCancer Immune Profiling panel. (A) Volcano plot showing the distribution of fold changes in gene expression in LH-EVOL challenged mice compared to AF293 challenged mice. (B) Pathway signature of mice challenged with either PBS, AF293, or LH-EVOL. Orange color indicates a pathway is enriched, while blue color indicates a pathway is decreased. (C) C57BL/6 and IL-1 $\alpha$ mice were inoculated with either $4 \times 10^{7}$ conidia of either AF293 or LH-EVOL. At 40 h postchallenge mice were euthanized and lungs saved for histological analysis. Formalin-fixed lungs were paraffin embedded, sectioned, and stained with GMS for microscopic analysis of fungal germination. Data are pooled from two independent experiments. Statistical significance was determined using a Mann-Whitney U test. 
(A)

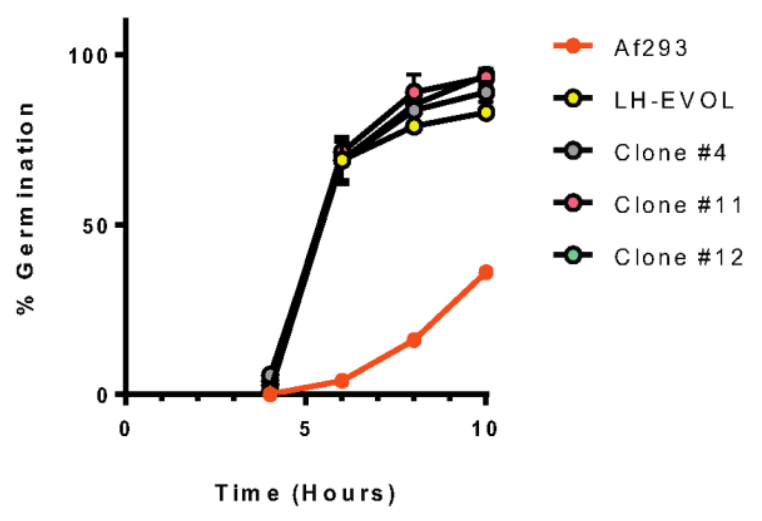

(B)

AF293 CCGACC-CCCCCCACTCA

Clone \#4 CCGACCCCCCCCCACTCA

Clone \#11 CCGACC--CCCCCACTCA

Clone \#12 CCGACC--CCCCCACTCA

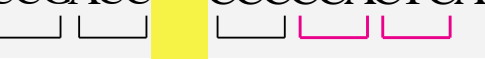

(C)

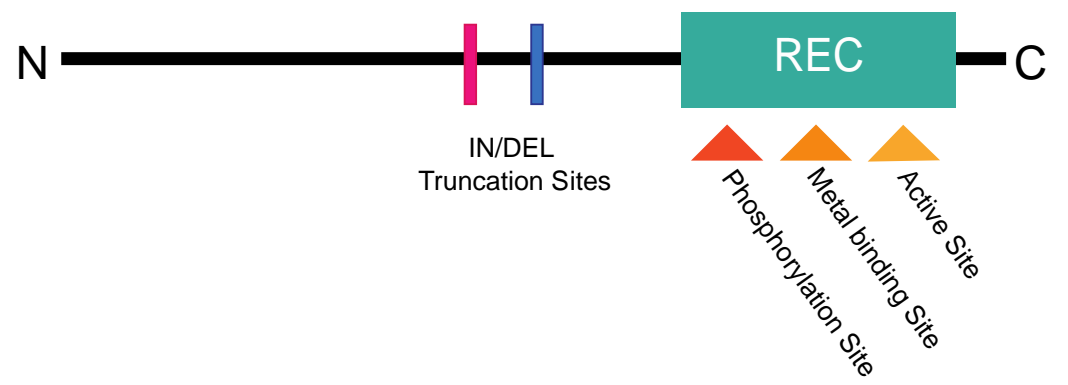

Figure 3. Identification of INDEL mutations in the Afu5g08390 (sskA) gene of A. fumigatus. (A) Percent germination over time in lung homogenate media of AF293, the bulk LH-EVOL strain, or individual LH-EVOL clones (clone \#4, clone \#11, or clone \#11). Data are representative of at least 2 independent experiments consisting of 3 biological replicates per group. Each symbol represents the group mean \pm 1 standard error of the mean. Statistical significance was determined using one-way ANOVA with Dunn's test. (B) DNA-sequencing of LH-EVOL clones \#4, \#11, and \#12 revealed INDEL mutations in the Afu5g08390 (sskA) gene. (C) Based on the predicted amino acid changes due to the frameshift caused by these INDEL mutations it is predicted that a premature stop codon causes the truncation of SskA prior to its REC domain in the LH-EVOL strains. 

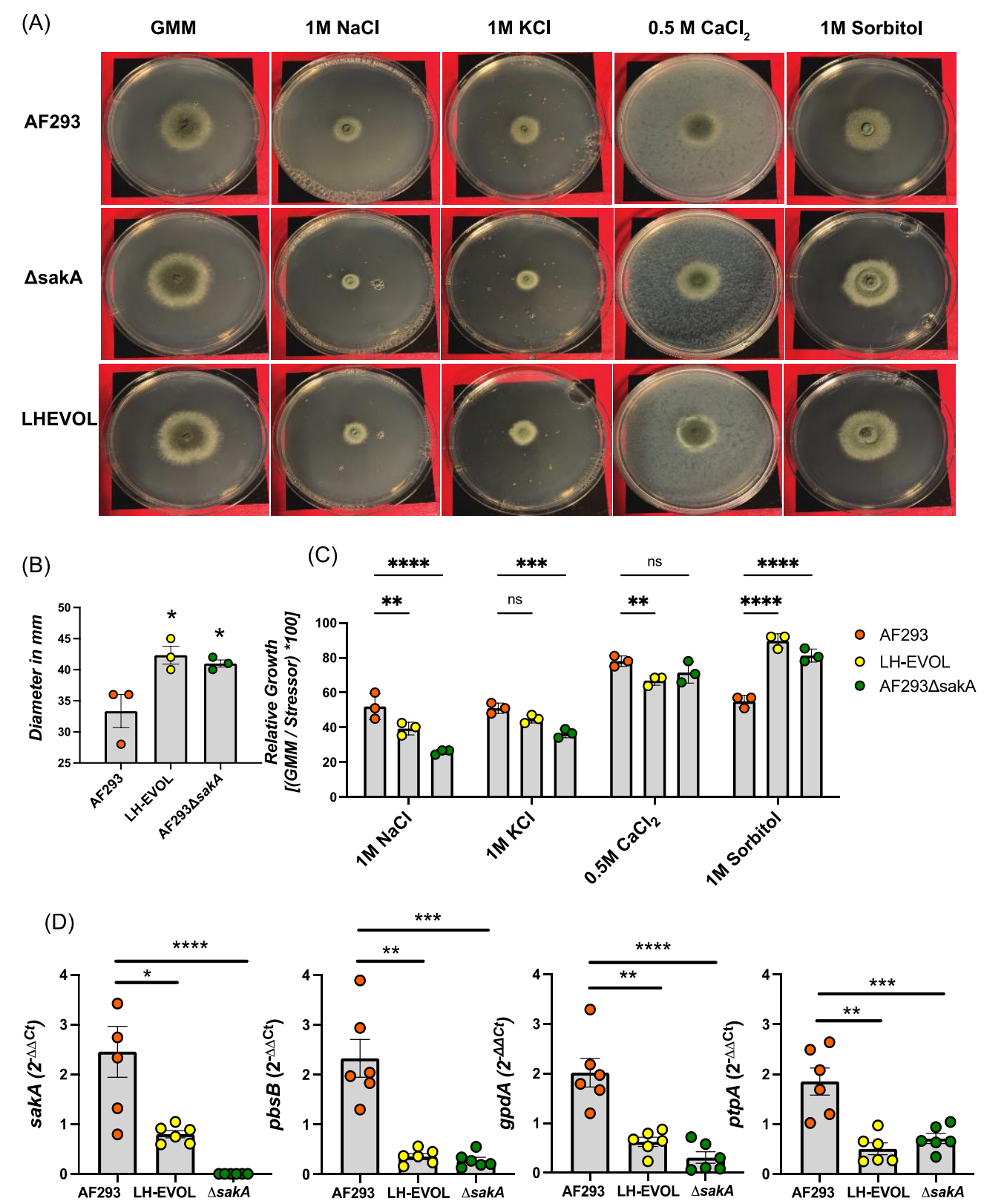

Figure 4. LH-EVOL strain demonstrates deficiencies in sakA-dependent osmotic stress response. (A) AF293, AF293 $\Delta s a k A$, and LH-EVOL conidia were inoculated on GMM plates or GMM plates supplemented with $1 \mathrm{M} \mathrm{NaCl}, 1 \mathrm{M} \mathrm{KCl}, 0.5 \mathrm{M} \mathrm{CaCl}_{2}$, or $1 \mathrm{M}$ Sorbitol and incubated for 72 hours at $37^{\circ} \mathrm{C}$. (B) Diameter of colonies grown on GMM for 72 hours in millimeters (mm). (C) Diameter of the colony was used to calculate the percent of inhibited growth compared to its respective measurement on GMM. (D) A 30-hour biofilm was transferred to fresh liquid GMM media supplemented with $1 \mathrm{M} \mathrm{NaCl}$ for 30 minutes at $37^{\circ} \mathrm{C}$. Quantitative RT-PCR was used to measure the change in transcript production of $s a k A$ and $s a k A$-dependent genes $p b s B, g p d A$, and $p t p A$. Ct values were initially adjusted to actA for $\Delta \mathrm{Ct}$, then $1 \mathrm{M} \mathrm{NaCl}$ was compared to GMM for $\Delta \Delta \mathrm{Ct}$ values. Data are pooled of results from 2 independent experiments. Statistical significance was assessed using (B) a one-way ANOVA with Tukey's test, (C) a two-way ANOVA and (D) one-way ANOVA with a Kruskal-Wallis test. $(*)$ indicates a $\mathrm{p}$-value $\leq 0.05$; $(* *)$ indicates a $\mathrm{p}$ value $\leq 0.01 ;(* * *)$ indicates a $\mathrm{p}$-value $\leq 0.001$. 

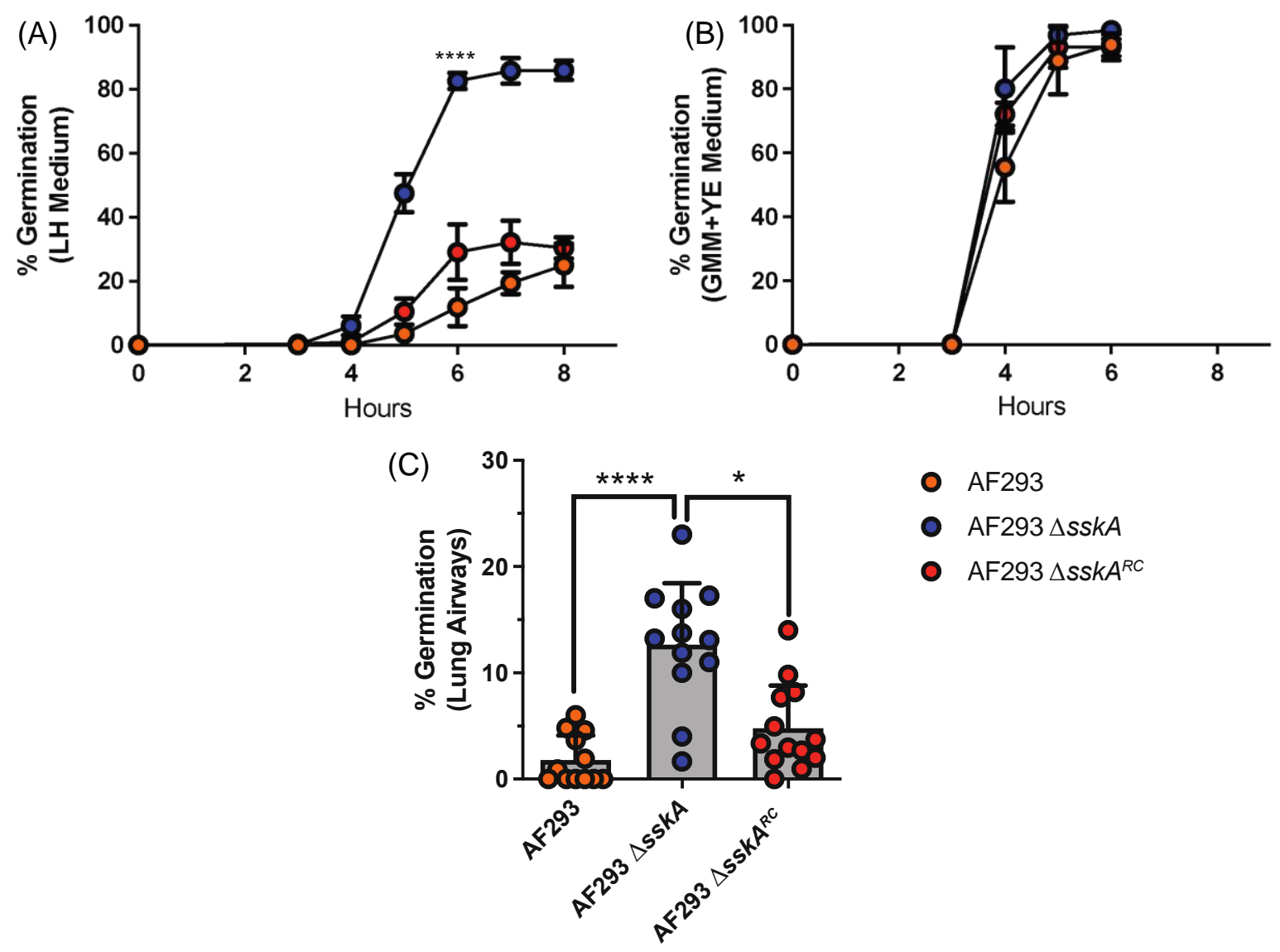

- AF293

- $\mathrm{AF} 293 \Delta s s k A$

- AF293 $\Delta s s k A^{R C}$

Figure 5. Loss of sskA in AF293 increases germination rate. Conidia germination of AF293, AF293 $\Delta s s k A$, and AF293 $\Delta s s k A^{R C}$ was determined by wet mount by microscopically counting the numbers of conidia and germlings in either lung homogenate media (A) or 1\% GMM supplemented with yeast extract (B). Data are representative of at least 2 independent experiments consisting of 3 biological replicates per group, Each symbol represents the group mean \pm 1 standard error of the mean. (C) In vivo conidia germination in airways of C57BL/6J mice with $4 \times 10^{7}$ conidia of AF293, AF293 $\Delta s s k A$, and AF293 $\Delta s s k A^{R C}$. At $12 \mathrm{hpi}$, mice were euthanized, and BALF was collected to quantify germination in the airways. Data are pooled of results from 2 independent experiments. Statistical significance was assessed using an (A) unpaired t-test or (C) one-way ANOVA with a Dunn post-test. $(*)$ indicates a p-value $\leq 0.05 ;(* *)$ indicates a $p$-value $\leq 0.001$. 
(A)

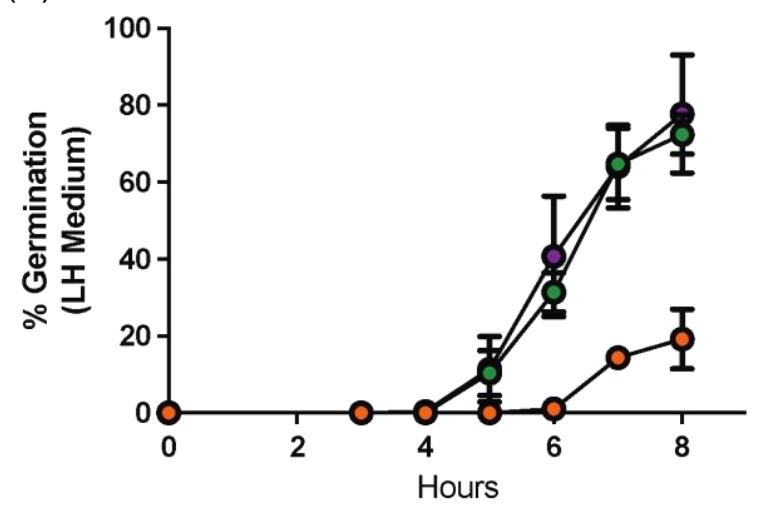

(B)

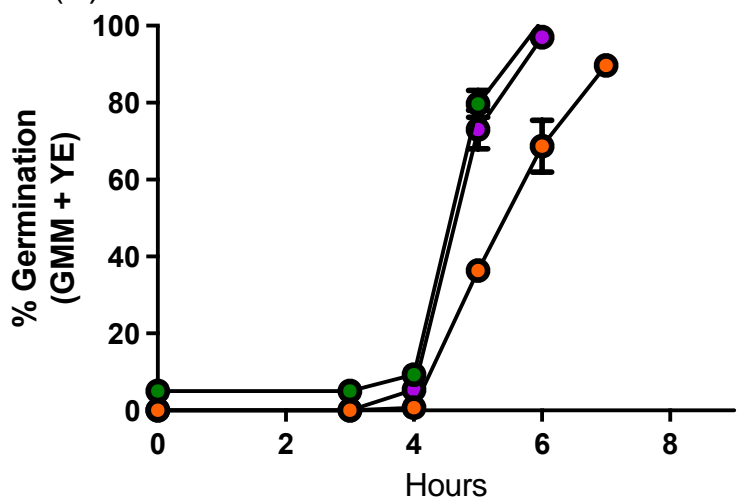

(C)

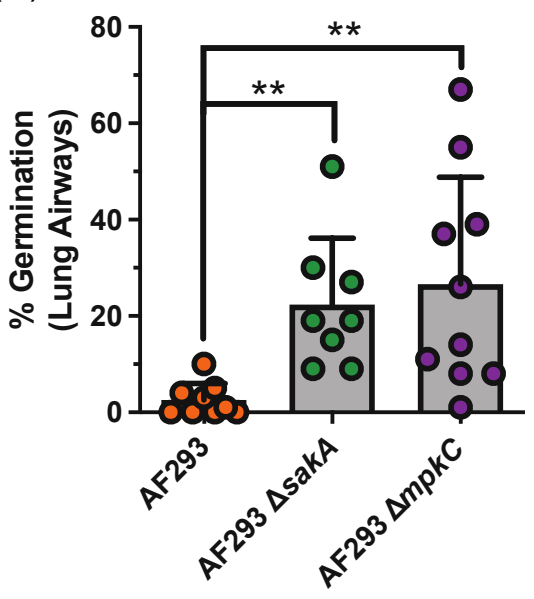

- AF293

- $\mathrm{AF} 293 \triangle \mathrm{sak} A$

- AF293 mpkC

Figure 6. SakA- and MpkC-null mutants have increased conidia germination kinetics in vitro and in vivo. (A-B) Conidia germination of AF293, AF293 $\Delta s a k A$, and AF293 $\Delta m p k C$ was determined by wet mount by microscopically counting the numbers of conidia and germlings in either lung homogenate media (A) or 1\% GMM supplemented with yeast extract (B). Data are representative of at least 2 independent experiments consisting of 3 biological replicates per group. Each symbol represents the group mean \pm 1 standard error of the mean. (C) In vivo conidia germination in airways of C57BL/6J mice with 4x107 conidia of AF293, AF293 $\Delta s a k A$, and AF293 $\triangle m p k C$. At $12 \mathrm{hpi}$, mice were euthanized, and BALF was collected to quantify germination in the airways. Data are pooled of results from 2 independent experiments. Statistical significance was assessed using an (A) unpaired t-test or (C) one-way ANOVA with a Dunn post-test. (**) indicates a $\mathrm{p}$-value $\leq 0.01$ 
(A)

AF293

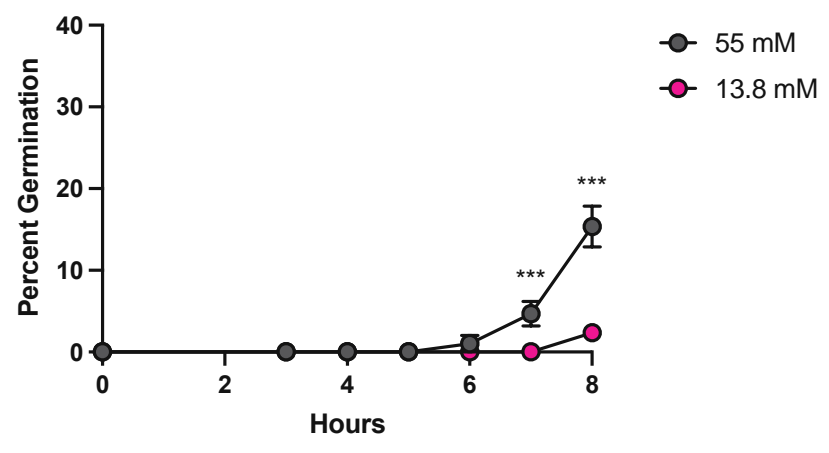

(B)

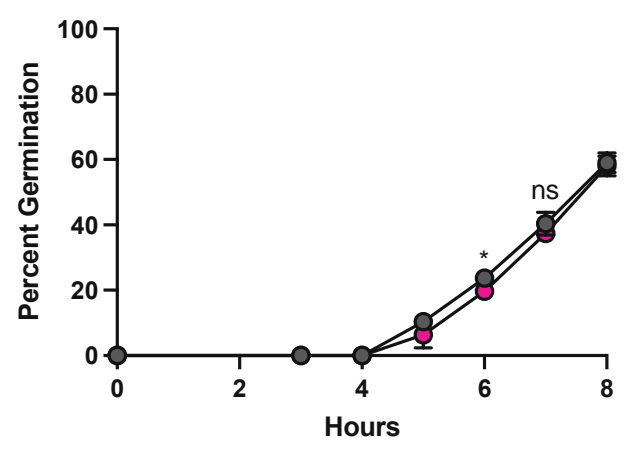

(D)

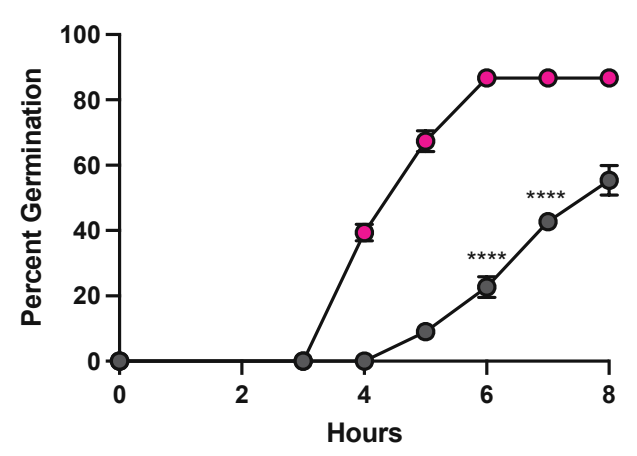

(C) AF293 $\triangle$ sakA

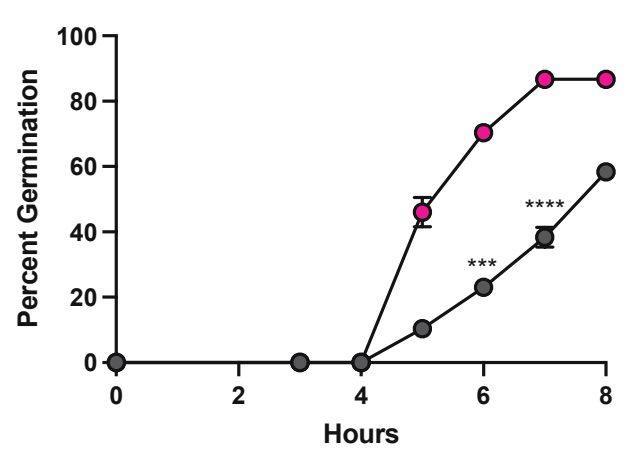

(E)

AF293 $\Delta m p k C$

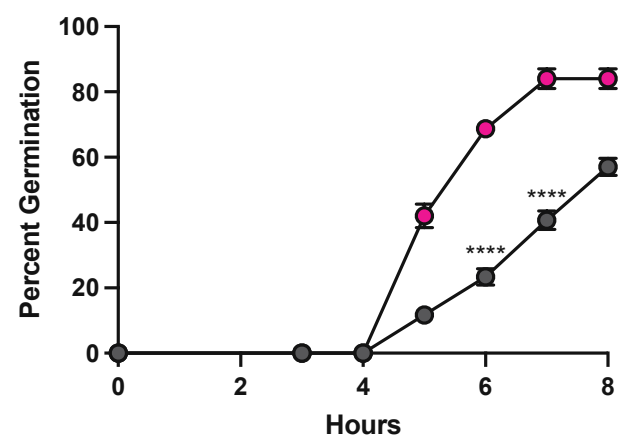

Figure 7. Increased germination rate of lung-adapted $A$. fumigatus strains in low glucose conditions. Conidia germination of AF293 (A), LH-EVOL (B), AF293 $\Delta s a k A$ (C), AF293 $\Delta s s k A$, (D) and AF293 $\triangle m p k C$ (E) was determined by wet mount and microscopically counting the numbers of conidia and germlings in either $1 \% \mathrm{GMM}$ or $0.25 \%$ GMM medium. Statistical significance was assessed using an unpaired Student's t-test. $(*)$ indicates a p-value $\leq 0.05 ;(* *)$ indicates a $\mathrm{p}$-value $\leq 0.01 ;(* * *)$ indicates a $\mathrm{p}$-value $\leq 0.001 ;(* * * *)$ indicates a $\mathrm{p}$-value $\leq 0.0001$. 
SUPPLEMENTAL TABLE 1. Strains used in this study. The strain identifiers, genotypes, and source information for all fungal strains included in this study.

\begin{tabular}{|l|l|l|}
\hline Strain ID & Genotype & Source \\
\hline AF293 & Reference Strain & Nierman et al. $2005^{68}$ \\
\hline CEA10 & Reference Strain & Girardin et al. $1993^{69}$ \\
\hline LH-EVOL & $\begin{array}{l}\text { Experimentally evolved from } \\
\text { AF293 }\end{array}$ & This study \\
\hline AF293 $\Delta s s k A$ & AF293 mutant lacking sskA & This study \\
\hline AF293 $\Delta s s k A^{R C}$ & $\begin{array}{l}\text { Ectopic complementation strain } \\
\text { of the AF293 } \Delta s k A \text { mutant }\end{array}$ & This study \\
\hline AF293 $\Delta s a k A$ & AF293 mutant lacking sakA & Xue et al. $2004^{13}$ \\
\hline AF293 $\Delta m p k C$ & AF293 mutant lacking $m p k A$ & Reyes et al. $2006^{52}$ \\
\hline
\end{tabular}


SUPPLEMENTAL TABLE 2. Primers used to generate AF293 $\Delta s s k A$ and AF293 $\Delta s s k A:: s s k A$.

\begin{tabular}{|c|c|}
\hline $\begin{array}{l}\text { Strain } \\
\text { ID/Purpose }\end{array}$ & Primers and Template DNA Information \\
\hline \multirow{5}{*}{$\operatorname{ssk} A^{R C}$} & Recon fragment from AF293 gDNA, PacI/NotI restriction site introduction \\
\hline & $\begin{array}{l}\text { 5'-AAAAAATTAATTAATGACTGGGTGGAAATGTTTTTTCAGAGAG-3' } \\
\text { 5'-AAAAAAGCGGCCGCTGGACGAATCAATAATGTGCGGGC-3' }\end{array}$ \\
\hline & $\begin{array}{l}\text { Real -time qPCR Primers for sskA } \\
\text { 5'- CAACCGTTCCCGAATTG-3' } \\
\text { 5'- GCTGGCTGGTGAATCT -3' }\end{array}$ \\
\hline & $\begin{array}{l}\text { Real-time qPCR Primers for } t u b 2 \\
\text { 5'- ATAATGTTCAGACCGCCCTCTGCT-3' } \\
\text { 5'- GACGGATGTGGAATTGCCCACAAA-3' }\end{array}$ \\
\hline & $\begin{array}{l}\text { Real-time qPCR Primers for } a c t A \\
\text { 5'- TCACTGCCCTTGCTCCCTCGTC-3' } \\
\text { 5'- G GCACTTGCGGTGAACGATCGAA-3' }\end{array}$ \\
\hline \multirow{4}{*}{$\Delta s s k A$} & $\begin{array}{l}\text { 5' Flank Amplification from AF293 gDNA, includes overhang for } p y r G \text { fusion } \\
\text { 5'-GCCTGGCGATAATGAGAGTCAG-3' } \\
\text { 5'-AGA GCA TTG TTT GAG GCG ACC } \\
\text { GGTCACAGTGCTTGACTGATAGCACAGC-3' }\end{array}$ \\
\hline & $\begin{array}{l}\text { 3' Flank Amplification from AF293 gDNA, includes overhang for } p y r G \text { fusion } \\
\text { 5'- CGCATCAGTGCCTCCTCTCAGACCCAGTTGCACTTTCTGCAAATCAGG-3' } \\
\text { 5'- CCGCATCAAGGATGTCGTTGTCAG-3' }\end{array}$ \\
\hline & $\begin{array}{l}\text { Aspergillus parasiticus pyrG gene from pSD38.1 } \\
\text { 5'- ACCGGTCGCCTCAAACAATGCTCT-3' } \\
\text { 5'- GTCTGAGAGGAGGCACTGATGCG-3' }\end{array}$ \\
\hline & $\begin{array}{l}\text { Fusion primers with 5' fragment, 3' fragment, and pyrG gene } \\
\text { 5'- TGACTGGGTGGAAATGTTTTTTCAGAGAG-3' } \\
\text { 5'- GCCCACTCTTTCTGACCCTGTTGA-3' }\end{array}$ \\
\hline
\end{tabular}


Supplemental Table 3. Primers used for transcriptional analysis of SakA-dependent genes.

\begin{tabular}{|l|l|l|}
\hline Name & Forward Sequence 5'-3' & Reverse Sequence 3'-5' \\
\hline$g p d A$ & ACGAGATCAAGCAGGCCATC & TCAGTGTAGCCGAGGATGTTC \\
\hline$p t p A$ & ACATTATGCCAGGGGACACA & GATCATTGGCACCGCATCTT \\
\hline$p b s B$ & GCCTGGCCGCAAAAAGAATG & AAGAGTCCCTGCCTTCGTGT \\
\hline sakA & CCGTGAGCTGAAACTGTTGA & GTCTGTCCCAAGGAGCTCTG \\
\hline$a c t A$ & AACTTGCGAGATAGCCTCCA & CCATGTCATCCCAGTTTGTG \\
\hline
\end{tabular}


Supplemental Table 4. Protein Sequences used in BLASTp analysis in non-redundant database, GenBank Accession.

\begin{tabular}{|l|l|l|l|}
\hline & Af293 & CEA10 & Similarity (\%) \\
\hline TcsB & XP_001481640.1 & EDP53731.1 & 97.87 \\
\hline YpdA & XP_751798.2 & EDP50401.1 & 100 \\
\hline SskA & XP_753797.1 & EDP51583.1 & 100 \\
\hline SskB & XP_752459.1 & EDP56327.1 & 100 \\
\hline PbsB & XP_752961.1 & EDP56828.1 & 100 \\
\hline SakA & XP_752664.1 & EDP56531.1 & 100 \\
\hline MpkC & XP_753727.2 & EDP51653.1 & 98.94 \\
\hline
\end{tabular}


A

Wild Type sskA locus

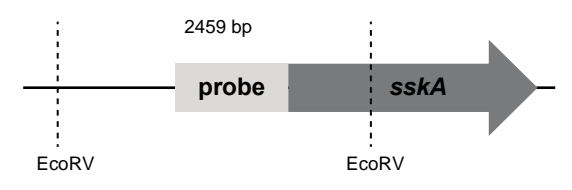

sskA::pyrG locus

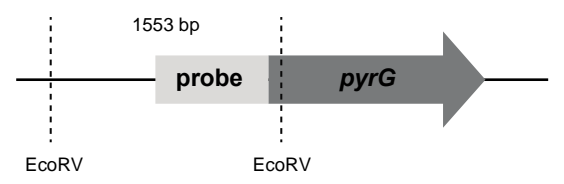

B

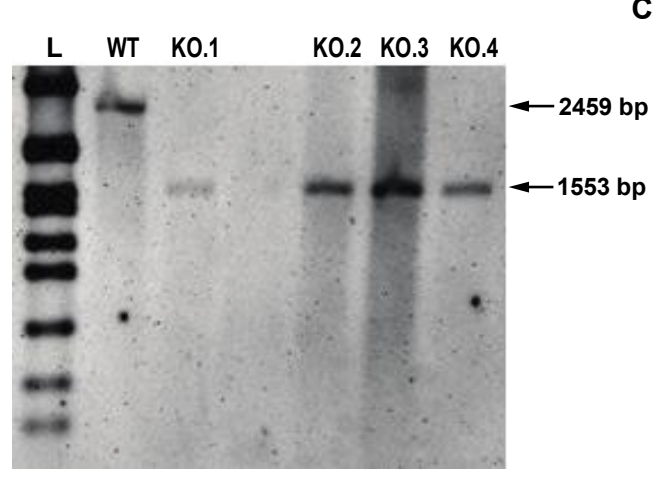

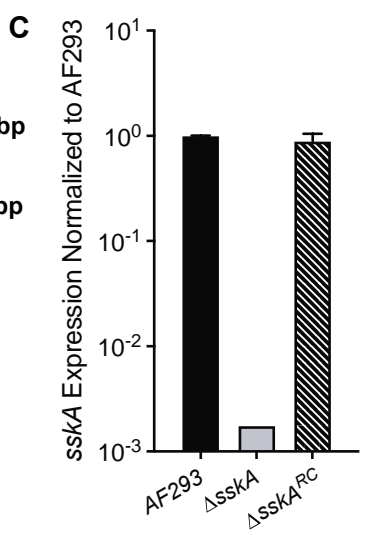

Supplemental Figure 1. Construction and confirmation of $A$. fumigatus $\Delta s s k$ and $\Delta s s k A^{\mathrm{RC}}$ strains. (A) Schematic of sskA gene replacement in A. fumigatus strain AF293.1 with expected band sizes following genomic DNA digestion with EcoRV. (B) Southern blot analysis of EcoRVdigested genomic DNA with a 1088 bp digoxigenin-labeled probe reveal successful gene replacement of $s s k$ with a single copy of pyrG in multiple transformants (KO.1, KO.2, KO.3, KO.4). (C) Real-time quantitative PCR results for sskA mRNA levels in the WT (AF293), $\Delta s s k A$ (KO.4), and $s s A^{\mathrm{RC}}$ strains from 18 hour liquid cultures. 
(A)
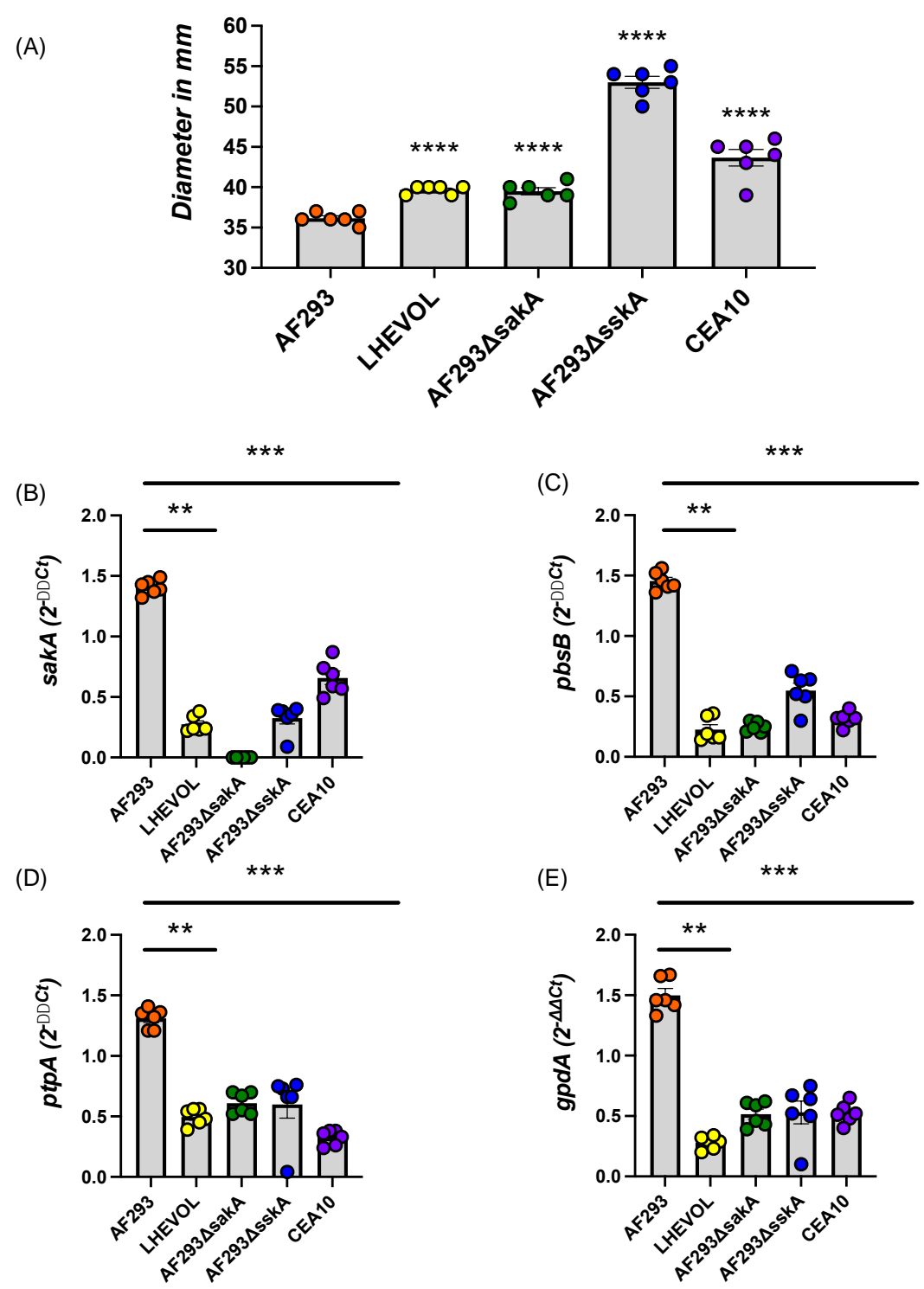

Supplemental Figure 2. CEA10 and AF293 $\Delta s s k A$ strain demonstrates deficiencies in sakAdependent osmotic stress response. (A) Diameter of colonies grown on GMM for 72 hours in millimeters (mm). (B-E) A 30-hour biofilm was transferred to fresh liquid GMM media supplemented with $1 \mathrm{M} \mathrm{NaCl}$ for 30 minutes at $37^{\circ} \mathrm{C}$. Quantitative RT-PCR was used to measure the change in transcript production of (B) $s a k A$ and $s a k A$-dependent genes (C) $p b s B$,(D) gpdA, and (E) $p t p A$. Ct values were initially adjusted to actA for delta $\mathrm{Ct}$, then $1 \mathrm{M} \mathrm{NaCl}$ was compared to GMM for delta delta $\mathrm{Ct}$ values. Data are pooled of results from 2 independent experiments. Statistical significance was assessed using (B) a one-way ANOVA with Tukey's test and (D) Mann-Whitney test and one-way ANOVA with a Kruskal-Wallis test. $(* *)$ indicates a p-value $\leq$ $0.01 ;(* * *)$ indicates a p-value $\leq 0.001 ;(* * * *)$ indicates a $p$-value $\leq 0.0001$. 
(A)

AF293 GDQHRILQVLINLVSNSLKFTPEHGKVEVRIKCVREAETPG
696

CEA10 GDQHRILQVLINL-----------VEVRIKCVREAETPG

(B)

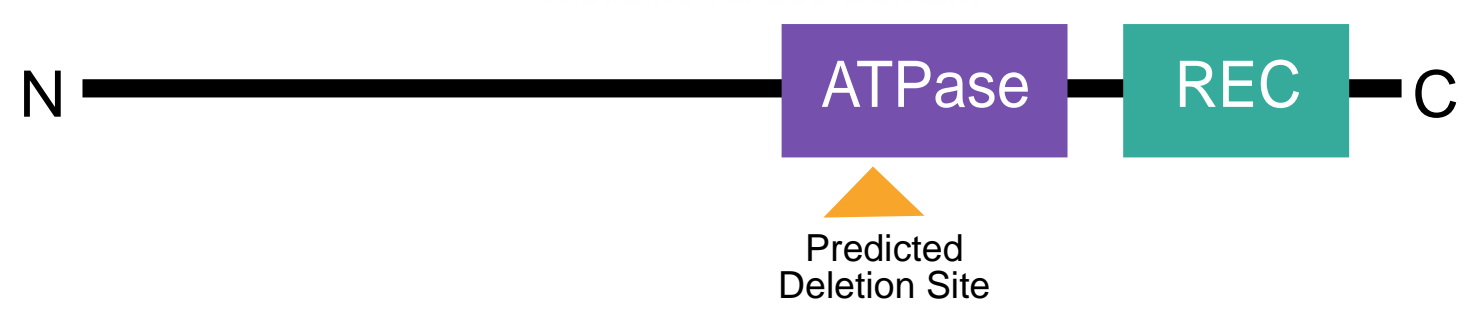

Supplemental Figure 3. Amino acid alignment of sensory histidine kinase response regulator TcsB in AF293 and CEA10. (A) Amino acid sequence demonstrating deletion (709-721, XP_001481640.1) within the predicted ATPase (696-863, XP_001481640.1) in the histidine kinase domain in CEA10 when compared to AF293. (B) Schematic of TcsB protein demonstrating relative position of amino acid deletion in CEA10. 